\title{
Photonic applications of azobenzene molecules embedded in amorphous polymer
}

\author{
S. De Martino ${ }^{1,2}$ (D) F. Mauro ${ }^{1,2} \cdot$ P. A. Netti ${ }^{1,2}$
}

Received: 23 October 2020 / Accepted: 2 January 2021 / Published online: 8 March 2021

(c) The Author(s) 2021

\begin{abstract}
The incorporation of azobenzene chromophores into polymer systems gives rise to a number of unique effects under UV and visible light irradiation. The light-driven isomerization of the azobenzene element acts as a light-to-mechanical energy converter, translating the nanoscopic structural movement of the isomerization azobenzene into macroscopic topographic film modulation in the form of surface relief. This review focuses on the study of reversible changes in shape in various systems incorporating azobenzene, including large-scale superficial photo-patterned glassy materials, light-driven reshaping of tridimensional superficial azo-textures and contractions of stimuli-responsive liquid crystalline networks (LCNs). Further, promising applications of azo systems are investigated as smart biointerfaces able to mimic time-varying biological systems.
\end{abstract}

Keywords Azobenzene $\cdot$ Azopolymers $\cdot$ Amorphous polymer $\cdot$ Photonic $\cdot$ SRG

\section{Introduction}

Azobenzene-type materials are constituted by an azo-moiety easily doped or covalently bound to a polymer matrix. The azo molecule was commonly used as dye and colorant until its unique optical properties were discovered and exploited for a large variety of applications. More precisely, when illuminated by UV and visible light, the molecule undergoes a reversible photoisomerization from trans to cis configuration, which affect local chemical, physical and mechanical properties of the host material. Illumination of an azomaterial with a linearly polarized light induces dichroism and

P. A. Netti

nettipa@unina.it; paolo.netti@iit.it

1 Center for Advanced Biomaterials for Healthcare, IIT@CRIB, Istituto Italiano di Tecnologia, Largo Barsanti e Matteucci, 53, 80125 Naples, Italy

2 Dipartimento di Ingegneria Chimica dei Materiali e della Produzione Industriale, DICMAPI, Interdisciplinary Research Centre On Biomaterials (CRIB), Università degli Studi di Napoli Federico II, Piazzale Tecchio, 80, 80125 Naples, Italy 
stable bulk birefringence due to molecule dipole transition moment reorientation perpendicularly to the light polarization. The anisotropic light induced birefringence can be optically erased by circularly polarized light or non-polarized light. In amorphous azopolymers, a continuous trans-cis-trans cycling of the azobenzene molecules under light illumination drives a photoinduced surface-pattern formation. In fact, a macroscopic mass transport can be observed on the surface of azo-aromatic polymer films as a consequence of the repeated light induced isomerization of the azo molecule, even below the glass transition temperature Tg of the polymer [1, 2]. Photo-induced azopolymer movement involves a reconstruction of the host material, inducing the modulation of the film thickness and the local stiffness, which results in the formation of surface relief gratings (SRG) on the polymer surface. The induced surface deformation profile appears dependent on the light intensity and polarization of the applied interference pattern. However, the mechanism underneath the mass migration process is still to be fully understood and is currently under investigation of the scientific community. As today, several models to describe the phenomena have been proposed [3-8]. Recently, directional light-induced mass migration phenomenon is exploited as a strategy for the fabrication of controlled tridimensional superficial textures. Indeed, azopolymer structures, such as an array of imprinted micropillars, can be deformed by means of the light-driven reconfiguration into complex configuration (e.g., mushroomlike structure) hardly obtainable by traditional techniques [9-14]. In addition, the azo compound has also been frequently coupled to liquid crystal systems (LC), where the light-induced azo molecule photoisomerization causes the isothermally LC-toisotropic phase transition of the mixtures, resulting in an order-disorder change of the system [15]. Photo-deformable liquid crystal polymers enable diverse deformations of materials along any chosen direction by using linearly polarized light. In these systems, light can be converted into mechanical work, resulting in large volume deformations such as contraction, expansion, bending or torsion. Azo LC systems dramatically increase the potentialities of LC systems combining their alignmentdependent shape-change with the azo mesogens that enable the remote control and diverse deformations of materials.

Along with the enormous diffusion of azopolymers in photonics and optical applications [15-21], a growing interest in cell interfaces research is emerging especially into the design of bioactive interfaces[22, 23]. Indeed, in the last years the innovative utilization of azopolymer materials in biological field has been widespread, since their light-responsive properties provide great advantages, including the preservation of the physiological conditions ( $\mathrm{pH}$, temperature) needed for living cells and the highly spatio-temporal control over several stimuli triggered by the azo molecule photoisomerization. Thus, the photopatterned surface of azopolymer films has been commonly used to provide topographic stimuli to cells in order to examine cell functions such as adhesion, spreading, alignment, migration and differentiation [24-30]. Furthermore, in the framework of biological response to physical environment, the cycling photoisomerization of azo molecules and the writing/erasure potentiality of azopolymer surface reliefs has been exploited in several works in order to provide dynamic signals to cells [12, 31-36].

In this review, recent advances of topographic film modulation in the form of surface reliefs, are reviewed along with the physical aspects characterizing the azo molecules 
photoisomerization, reorientation and photomechanical effect concerning mass migration. In particular, the mechanism and intensity of photo-driven bulk movement and their dependency on the light source and polymer nature are discussed. An overview of the large range of applications of these molecules deriving from their inclusion into host amorphous or LC materials are reported. Finally, the innovative applications of these systems in the biological field as cell dynamic interfaces are discussed.

\section{Azobenzene photoswitches}

\subsection{Isomerization of azobenzene}

The molecular structure of azobenzene is characterized by two phenyl rings held together by the azo-linkage $(-\mathrm{N}=\mathrm{N}-)$. Isomerization of the azobenzene between the thermodynamically stable trans-form and the meta-stable cis-form occurs upon the absorption of a photon within its absorption band and involves changes in geometry and polarity of the molecule, which passes from the planar spatial configuration in the trans isomer to the non-planar in the cis isomer, thus resulting in a reduction of the distance between carbon atoms in position 4 in the aromatic rings from 9.9 to $5.5 \AA$ and an increase in the dipolar moment from 0.5 to 3D (Fig. 1a) [37, 38]. Trans azobenzene shows a $n-\pi^{*}$ band at $440 \mathrm{~nm}$ and a strong $\pi-\pi^{*}$ transition near $320 \mathrm{~nm}$. Cis azobenzene adopts a bent conformation and has a dipolar moment different from zero. It also has a strong $n-\pi^{*}$ band near $440 \mathrm{~nm}$ and shorter wavelengths bands at $280 \mathrm{~nm}$ and $250 \mathrm{~nm}$. The photoisomerization of the azobenzene molecule from trans to cis isomer upon UV/visible light irradiation is a reversible process in which the cis isomer can be back-isomerized to the trans form by thermal relaxation or by a new illumination in the cis-absorption band. These photoisomerization reactions occur on a timescale of picoseconds, while cis-population lifetime in the dark is typically on the order of hours, minutes and seconds depending on the substitution configuration [39]. Indeed, Rau [40] distinguished three classes of azobenzene characterized by the substitution of functional groups in the chemical configuration of the phenyl rings (Fig. 1b), which defines the different absorption spectra and photophysical response: the azobenzene-type molecules, the aminoazobenzene type, and the pseudo-stilbenes. Azobenzene-type molecules are similar to the parent (unsubstituted) azobenzene molecule with a low-intensity $n-\pi *$ band in the visible region and a much stronger $\pi-\pi^{*}$ band in the UV and with a cis lifetime of days. The aminoazobenzene type molecules which are ortho or para substituted with an electron-donating group, with the $n-\pi^{*}$ and $\pi-\pi^{*}$ bands much closer, exhibit absorption band in the visible blue region and are characterized by an intermediate lifetime in the dark. Pseudo stilbenes, which are substituted at 4 and $4^{\prime}$ positions with an electron-donating group and electron withdrawing group, have red-shifted absorption band and a very rapid cis-to-trans reconfiguration since their absorption spectra overlap. Among them, pseudo stilbenes have the best photoswitching properties, making them the most used azobenzene type molecules. Their strong $\pi \rightarrow \pi^{*}$ absorption with charge-transfer (CT) character is a dominant feature in the visible spectrum, and $n \rightarrow \pi^{*}$ transition is hidden by this strong band. Substituents on the phenyl rings may strongly influence the position and shapes 
A

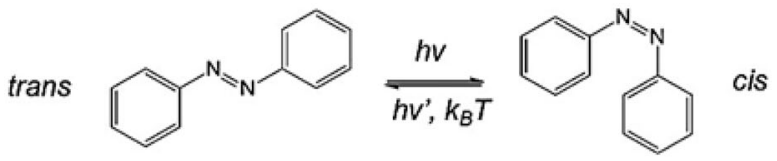

B<smiles>c1ccc(N=Nc2ccccc2)cc1</smiles>

Azobenzene<smiles>Nc1ccc(N=Nc2ccccc2)cc1</smiles>

Aminoazobenzene<smiles>Nc1ccc(N=Nc2ccc([N+](=O)[O-])cc2)cc1</smiles>

Pseudo-stilbene

\section{blue shifted days}

C

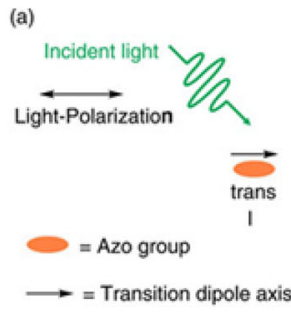

$\lambda_{\max }$

$\tau_{1 / 2}$

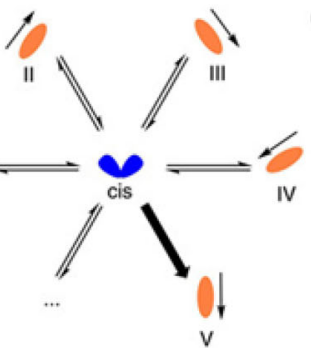

(b)

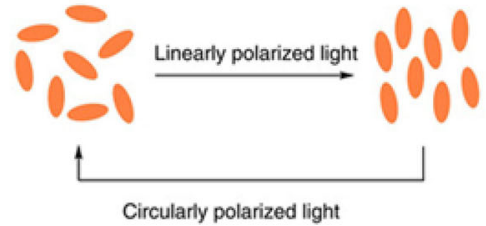

Fig. 1 a Chemical structure of azobenzene. Trans-cis photoisomerization upon light irradiation and cis-trans backward reaction by thermal relaxation or optical illumination (Reproduced with permission from Ref. [41], Copyright (C) 2013 Wiley Periodicals, Inc.). b The three classes of azobenzene and their photophysical properties (Reproduced with permission from Ref. [41], Copyright (C 2013 Wiley Periodicals, Inc.). c The trans isomer oriented in the direction of light polarization will absorb the incident light and photo-isomerize assuming a new random orientation. The molecules oriented perpendicularly will remain inert to the light. Multiple switches will result in the alignment of the trans population in the direction perpendicular to light polarization. The initial isotropic condition can be restored by circularly polarized light (Reproduced with permission from Ref. [66]. (c) 2018 Wiley-VCH Verlag GmbH \& Co. KGaA, Weinheim)

of the azobenzene absorption bands and the rate of thermal cis-to trans relaxation [39, 41].

\subsection{Azobenzene photo orientation and birefringence}

When azobenzene chromophores are incorporated within a polymer matrix, continuous photoisomerization between the two isomeric states by polarized light can lead to a series of motion occurring at molecular, nanoscale and micrometer (macroscopic) levels [2]. This molecular motion is a statistical process, in which only azobenzene molecules with a transition dipole axis along light polarization direction will absorb the incident light and then photo-isomerize (Fig. 1c) [66]. More precisely, the probability of photon absorption varies as $\cos 2 \phi$, where $\phi$ is the angle between the light polarization and the azo dipole axis [39]. Therefore, azo molecules with dipole axis parallel to the electrical field will absorb and reorient in a new random direction, 
whereas those oriented perpendicularly will not move. After many cycles of photoisomerization, the azo chromophore will be stochastically oriented orthogonal to light polarization, resulting in a net reduction of the population of trans isomers aligned in the direction of polarization, with a concomitant increase of trans molecules oriented orthogonally. This process is referred to as orientation hole-burning [9]. However, the photo-orientation is reversible and the initial condition can be restored by using circularly polarized light [2]. This statistical reorientation gives rise to very strong birefringence (anisotropy in refractive index) and dichroism (anisotropy in absorption spectrum) [39].

The photoinduced birefringence is related to the structure of the azo chromophore, the type of the polymer backbone, and the interaction between azo dipoles [42]. Brown et al. observed to what extent the dipolar interaction between neighboring azo groups affect the level of photoinduced birefringence [43]. In poly(DR1A-co-MMA) and poly(DR1M-co-MMA) copolymers, the local electric field of neighboring dipoles raises or lowers the absorption energy of an azo group [43]. In more details, parallel dipoles decrease the absorption energy while antiparallel dipoles increase the absorption energy. At high azo contents, the dipoles prefer to align in an antiparallel fashion, which lowers the mobility of azo groups and, therefore, the induced birefringence value. In the lower azo content copolymers, the antiparallel pairing is not prevalent since the azo dipoles are isolated by MMA units, so that the induced birefringence per dipole becomes higher [43]. Further, the induced birefringence level can be higher for some semicrystalline polymers than amorphous polymers with comparable azo contents. It is due to cooperative motion of some photo inactive groups present and to the formation of semicrystalline domains [43].

Examples of cooperative motion have also been reported in side chain liquid crystalline copolymers. In this case, the effect of birefringence is amplifying over the limits given by the azo groups concentration, if a mixture of mesogens and non-photoactive mesogens was present in the side chains, these latter undergo a reorientation together with the azo mesogens, thus increasing the effect [42]. This phenomenon was known for side-chain polymers where the "inert" rigid groups had a similar shape and position in the molecule as the photochemical azobenzene group. Based on these properties, azobenzene-containing materials have been proposed as a platform for reversible data storage and holographic recording [43-46].

Further, azobenzene derivatives show strong photochromism i.e., photomodification of color [47]. This phenomenon is known as photoinduced optical anisotropy (POA) and can be detected in dichroism and birefringence measurements. Neporent and Stolbova [48] described POA in viscous solutions of azo dyes, then Todorov and co-workers [45] disclosed the same phenomenon in azo dye-polymer blends. The anisotropy induced in these systems is rather unstable, but the stability can be improved in azopolymers [49]. It turned out that stable anisotropy can be induced in both amorphous and liquid crystalline (LC) azopolymers [50-54]. Moreover, Nikolova et al. reported for the first time the possibility to induce a large circular anisotropy-circular dichroism and optical activity, in previously unoriented films of a liquid crystalline side-chain azobenzene polyester on illumination with circularly polarized light [55]. They stated that the photoinduced circular anisotropy was due to a new chiral phase, initiated by light-induced reorientation of the azobenzene chromophores as well as 
by the transfer of circular momentum from CPL to the azobenzenes, which adopt a cholesteric-like structure [56]. In addition, chiroptical properties photomodulation can be induced in polymers bearing azoaromatic chromophore linked to the side-chain or main-chain of the polymer backbone showing chiral conformations. Angiolini et al. investigated the photoinduced optical properties of a new class of methacrylic polymers bearing in the side-chain an optically active (S)-pyrrolidinyl ring directly linked through the nitrogen atom to a trans azo aromatic system substituted in the 4 position by an electron withdrawing group, such as cyano or nitro. These polymers exhibit reversible linear dichroism and birefringence when irradiated with linearly polarized (LP) light, but when illuminated with left circularly polarized (CP-L) light at $488 \mathrm{~nm}$, the circular dichroism (CD) spectra of the films show a net sign inversion that persists for at least several months at room temperature. The effect is reversible and the original shape can be restored by pumping with right circularly polarized (CP-R) radiation [57]. Also, Barberà et al. observed the same phenomenon in a chiral liquid-crystalline polymers containing the L-lactic residue of one absolute configuration in the side-chain [58]. It is worth to underline that these systems are chiroptical switches showing interconversion between enantiomeric chiral organizations in a reversible manner using orthogonal CPL suitable for applications in optical storage or chiral sensors [56].

\subsection{Azopolymer photomechanics}

The microscopic movements of the polymer chain, as a consequence of azo molecule photoisomerization, promote a macroscopic mass transport at the surface of polymer thin film at temperatures far below the polymers glass transition temperatures $\mathrm{Tg}$. The origin of macroscopic mass transport of the azopolymer matrix remains unclear. Nevertheless, several theories have emerged in order to shed light on this intriguing mechanism [59-61].

In polymers containing pseudo-stilbene azobenzene-type as side chains, the cyclic isomerization of azobenzenes involving the polymer chains induces a massive movement of the polymer material. The photo-isomerization of azobenzene molecules from a stable trans-state to a meta-stable cis-state during UV irradiation is an indispensable prerequisite for the polymer film deformation, but meanwhile, the mechanism of light induced polymer deformation is still debated [62-64].

Saphiannikova and coworker proposed a re-orientation model, assuming that the light-induced re-orientation of the azobenzene side chains induces a re-organization of polymer backbone caused by the appearance of a mechanical stress which in turn generate a mass migration [62]. This theory assumes that the material remains in the solid state during photoirradiation, and it is described with the term "moving" or "mass transport". The Karageorgiev's group, instead, supports an isothermal directional photo-fluidization model claiming that the cyclic isomerization of azobenzenes may cause an isothermal from an isotropic solid to an anisotropic liquid [63]. They findings support the viscoelastic model in which the light makes the molecules mobile in general and allows small forces to generate material flow. Another possible mechanism, which explains photoinduced liquefaction, is that trans-cis-trans cycles decrease $\mathrm{Tg}$ of azopolymers and drive azopolymers to 'flow' [65, 66]. However, light-reduced Tg 
does not have direct experimental proof. Pellerin and co-workers mentioned the localized photoplasticization, which can be associated with a free volume gradient, helps to understand photoinduced macroscopic flow far below $\mathrm{Tg}$ [67]. However, there are still contradictions about the mechanism and there is no overall theory which can describe all the findings.

Furthermore, also in azobenzene with a long lifetime in the cis form, it is reported a photoinduced liquefaction of azopolymers defined as photoinduced reversible solid-toliquid transitions [66]. This photoinduced solid-to-liquid transition based on trans-tocis isomerization is different from photoinduced liquefaction based on trans-cis-trans cycling. In this case, the photoinduced flow is non-directional and associated to a cyclic isomerization, but the mechanism for photoinduced solid-to-liquid transition of azopolymers is due to different Tg values of trans and cis azopolymers. In fact, it is reported that light can switch Tg of azopolymers and convert a trans azopolymer, that is solid, into a cis azopolymer (liquid), and further, the cis azopolymer stands in the liquid state even when UV light is switched off $[66,68]$. The light-induced mass migration phenomenon translates into the appearance of topographic surface reliefs, whose structural features depend on the intensity and polarization distributions of the irradiating light.

\section{Light-driven motion of azobenzene-containing materials}

The incorporation of azo chromophores in a large variety of materials represents an uncomplicated and versatile tool to offer them efficient photo-responsive properties [39]. Indeed, azo molecule photoisomerization upon light illumination leads to large movement occurring at microscopic scale, which generate macroscopic variations in the chemical and physical properties of the surrounding. These macroscopic phenomena have numerous implications in the technological area for the development of new materials characterized by light-matter interaction. Moreover, the possibility to modify the photochemical properties of azobenzenes through various synthetical routes allows to design materials with specific and tunable chemical, physical and mechanical characteristics. Recently, also supramolecular interactions (hydrogen, halogen and ionic bonds) between the azo molecule and the host material, were reported to have advantageous properties in terms of photoinduced anisotropy and surface patterning [70].

In 1984, Todorov et al.[71] first reported an optical anisotropy related to the photoisomerization of an azo dye dispersed into a polymer matrix when exposed to linearly polarized light (LPL). Later on, the same phenomenon was reported in LC [72] and amorphous [73] side-chain azopolymers, demonstrating the potential application of these materials. In 1995, Natansohn's [1] and Trypathy's [74] groups independently reported for the first time the formation of stable and highly efficient surface gratings optically induced on azopolymer film using interference irradiation of two laser beams. Periodic surface corrugation pattern called surface relief grating (SRG), was reported in various azobenzene functionalized polymers such as amorphous polymers [75-77], side chain liquid crystalline polymers [78, 79], supramolecular systems [80], amorphous molecular materials [81]. 


\subsection{Photo-patterned azomaterial surfaces}

The patterning process of azomaterial surfaces in response to light can be achieved by several techniques aiming at applying specific light intensity and polarization distributions depending on the application. Although the most common method to create surface relief gratings is the interference lithography in Lloyd's mirror configuration, other systems can be employed to induce the light-driven mass movement. Here, it is reviewed several scenarios in which regular surface reliefs or randomly-distributed surface reliefs were generated by different irradiation types, e.g., holographic light irradiation, 1D Gaussian laser beam and one-beam irradiation.

\subsubsection{Regular surface reliefs by holographic light irradiation}

As introduced, the first observation of spontaneous surface deformations on azopolymer film after light irradiation through an interference pattern occurred in 1995 by Natansohn's [1] and Trypathy's [74] groups. Azobenzene containing polymer films irradiated with optical interference patterns deforms into a wave-like, sinusoidal manner to form a surface relief grating. These sinusoidal surface patterning, known as SRG replicate the intensity or polarization distribution of the incident light. Kim et al. reported, for the first time, about the large scale periodic modulations of the free surface of a thin amorphous azobenzene-containing polymer film. It was irradiated with the sinusoidal intensity light pattern produced by the interference of two mutually coherent p-polarized laser beams [74] by a Lloyd mirror. It is the set-up used by original investigation in this area and it generates an interference pattern caused by intersecting two coherent laser beams with a wavelength in the azo absorption band. Figure $2 \mathrm{a}, \mathrm{b}$ shows a representative set-up example of SRG formation and a typical grating profile of a film surface after exposure to the laser. The surface grating depth can be controlled by adjusting the light exposure time, the polarization state of the beam and the angle of inscription $(\theta)$ [69] obtaining grating depths from few hundred nanometers to micron-deep gratings [3, 69]. In amorphous azopolymers (as opposed to LC azopolymers), efficient photoinduced surface-pattern formation is thought to be driven by continuous trans-cis-trans cycling of the azo benzene molecules [82], which align perpendicularly to the light polarization, inducing mass transport well below the material glass transition temperature $(\mathrm{Tg})$. Hurduc et al. directly observed the fluid state formation by optical microscopy during the SRG formation. They highlighted the importance of polymer chemical structure in the softening/fluidization [83] and divided the SRG formation in three different processes that can take place simultaneously: the polymer photo-fluidization in illuminated regions; the mass displacement from illuminated to dark regions and the inverse mass displacement from dark to illuminated regions (Fig. 2c).

The inscribed SRG topography is stable over years at room temperature and can be erased, recovering the primary flat surface, only by further intense treatment such as either irradiation with a circularly polarized beam or by heating the polymer sample above the glass transition temperature [2]. After the erasing, the azo films show no evidence of degradation or charring of the polymer films, concluding that light induced reversible structuring of photosensitive polymer films. The reversible nature 

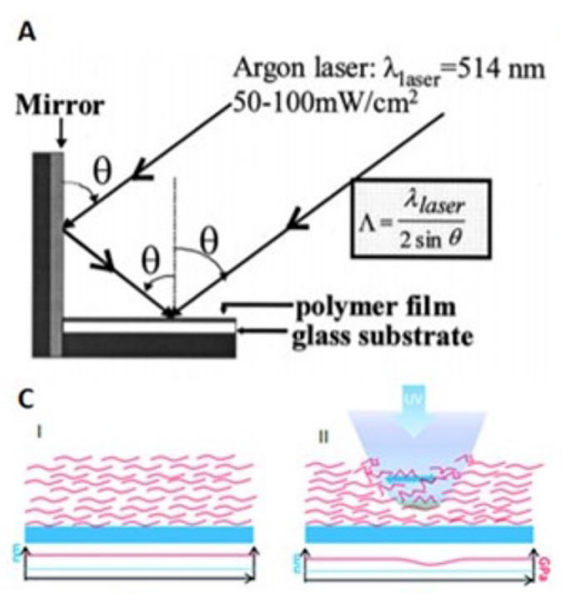

B

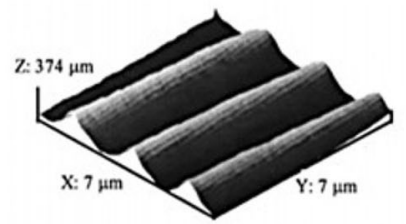

D

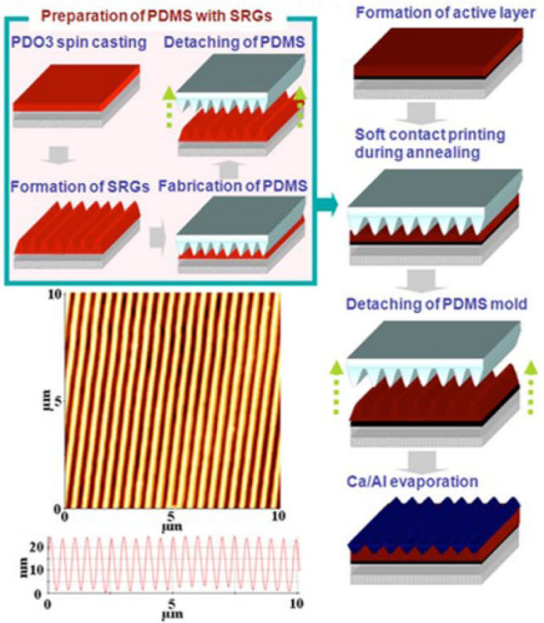

Fig. 2 a Optical setup for grating inscription. (Reprinted from Ref. [69] with the permission of AIP). b AFM surface profile of an optically inscribed grating in a pDR1M film (Reprinted from Ref. [69] with the permission of AIP). $\mathbf{c}$ The proposed mechanism for SRG formation during laser irradiation, involving at least three processes: (II) the polymer photo-fluidization in illuminated regions, (III) the mass displacement from illuminated to dark regions and (IV) the inverse mass displacement from dark to illuminated regions. (Republished with permission of Royal Society of Chemistry from Ref. [83]; permission conveyed through Copyright Clearance Center, Inc.). d Fabrication of organic solar cells using azopolymer SRGs. Creation of a PDMS stamp with the help of an SRG mold. The obtained PDMS stamp. The steps of fabrication of a solar cell using the created stamp (Reprinted from Ref. [103]. with the permission of AIP)

and its easily tunable making the SRGs attractive micro-texture pattern for numerous applications, such as optics [61, 84], photonics [82, 85] as Bragg reflectors used as light couplers and spectral filter [86-90], optoelectronic devices [91, 92], recorder holograms [93] and biological optics [94]. The patterned SRGs can be also used as an easy and cost-effective fabrication framework of large-scale periodic patterned with controlled anisotropic wettability [95, 96]. The degree of wetting anisotropy can be easily controlled through the irradiation parameters in the light-induced SRG inscription process. The texture depends explicitly on the modulation depth and on the periodicity of the rough surface [96]. Especially related to the design of optical devices, the structured azomaterial films are used directly as obtained after the light structuring exposure. For other applications, the direct use of a layer of photosensitive azomaterial can deteriorate structural stability as a consequence of undesired mass migration effects. In these cases, the SRGs can be used as molding templates and lithographic masks for the transfer of the superficial texture on other materials [97, 98] by a replica molding process [97, 99, 100]. These elastomeric stamps, made of poly(dimetylsiloxane) (PDMS), allow the reproduction of the complementary super- 
ficial SRG texture with high fidelity. Obviously, the pattern formation and quality depend on the factors such as the solution concentration, contacting time in the printing process, and printing pressure [97]. The easiness of master preparation of SRGs templates and the versatility of soft fabrication processes can be applied to the fabrications of optical functional surfaces, sensors, and photonic devices, organic solar cells [101-104] and organic light-emitting diodes (OLEDs) [105] (Fig. 2d) [16]. Furthermore, in recent years, azopolymer-based patterns have also been increasingly used as lithographic masks for fabricating periodic arrays of, e.g., titanium dioxide [106], indium tin oxide [98], and metallic [107] nanostructures.

\subsubsection{Regular surface reliefs by 1D Gaussian laser beam}

Although interference pattern configuration is commonly used to produce surface relief gratings on azopolymer films, a single focused laser beam can also be used to emboss surface gratings. Bian et al. reported a single Gaussian beam-induced deformation on different azopolymer materials [108]. They demonstrated that the presence of an intensity gradient by itself does not lead to surface deformation unless there is an optical field component present in that direction [108, 109]. This leads to a highly directional mass movement, with the polarization direction constituting a preferential direction along which the material transport mainly occurs [9]. The focused beam is able to make a hole in correspondence to the center of the laser spot and the resulting surface deformation is influenced by the polarization and intensity of the laser beam. A dip is formed in the center while the polymer chains move out from the central region and pile up at the wings where the intensity is small, when the surface deformation is induced by a linearly polarized Gaussian beam focused on an azopolymer film (Fig. 3a) [109]. In contrast, the polymer surface deformation results completely differently when induced by a higher-intensity linearly polarized Gaussian laser beam. In this case, a peak in the surface profile appeared in the center of the exposed spot (Fig. 3b) [108, 109]. This behavior at higher intensities can be explained by some photochemical reactions in addition to photothermal and photobleaching effects that can occur in polymeric matrix [109]. When the surface deformation is induced by a circular polarization Gaussian beam, the polymer moves from the center to the outside of the focused laser spot, thus forming a doughnut-shaped pattern (Fig. 3c). Moreover, a cylindrical Gaussian along the direction of the light intensity gradient (Fig. 3d) beam can induce the formation of a symmetric groove with the central bottom of the groove corresponding to the position of maximum light intensity. On the other hand, no appreciable deformation was observed when the laser beam had a polarization perpendicular to the direction of the light intensity gradient [109]. Therefore, the direction of the intensity gradient should match the direction of the linear light polarization in order to achieve a highly efficient surface structuration [9]. A direct observation of the directional azomaterial movement in the light polarization of a linearly polarized single focused beam was performed by Yadavalli et al., where they mechanically scratched the surface of a flat azopolymer film creating rectangular pieces, which were illuminated with a linear polarized single focused beam. As a consequence of the irradiation, the grooves orthogonal to the light polarization were closed, while the parallel grooves became larger (Fig. 3e) [64]. For all kinds of polarization of the Gaussian beam, the 
A

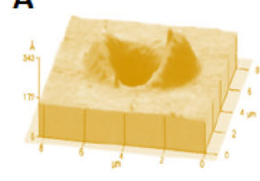

C

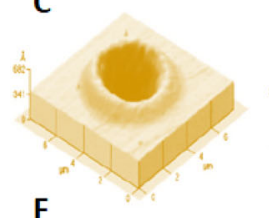

$0 \mathrm{~nm}$
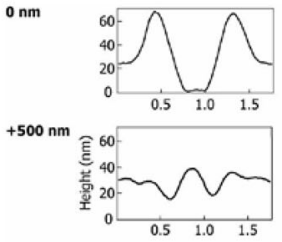

$-500 \mathrm{~nm}$

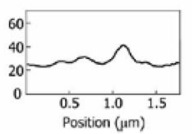

B

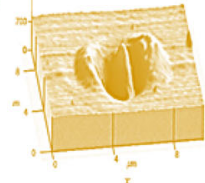

D
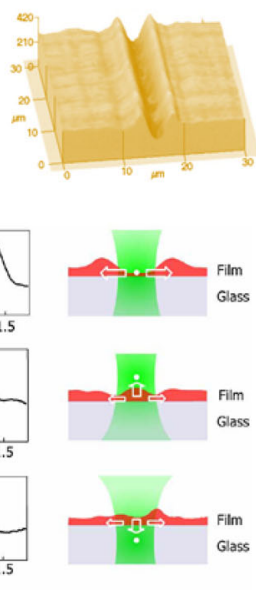
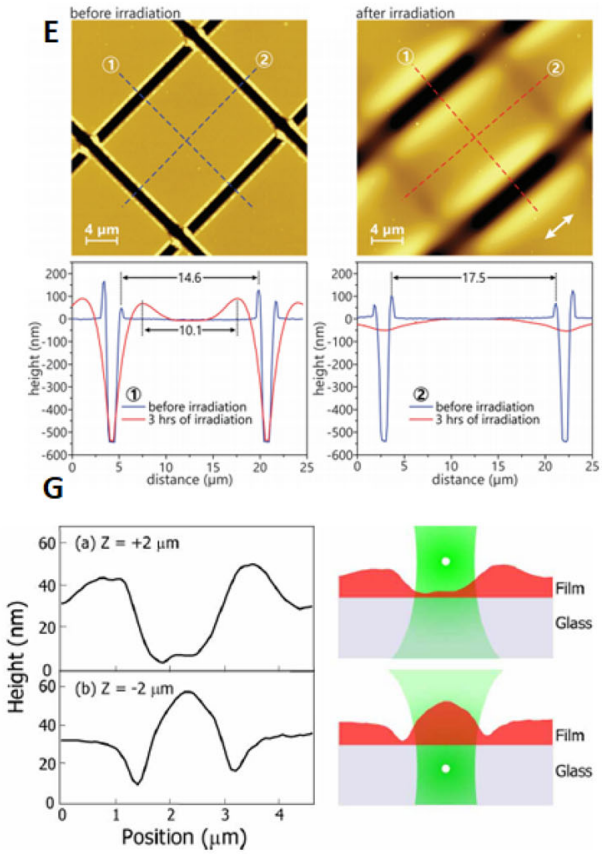

Fig. 3 a Surface deformation induced by Linear polarization Gaussian beams with low intensity and $\mathbf{b}$ with high intensity. c Surface deformation induced by circular polarization Gaussian beams with low intensity. d Surface deformation induced by a one-dimensional Gaussian beam (Reprinted from Refs. [108, 109], with the permission of AIP Publishing). e Polarization dependent mass-transport of Azo-PSI and AFM crosssectional analysis of the rectangular width, before and after irradiation. The direction of the polarization is marked by the white arrow (Republished with permission of Royal Society of Chemistry from Ref. [64]; permission conveyed through Copyright Clearance Center, Inc.). $\mathbf{f}$ Line plots of the surface deformation and schematics of the relationship between the $Z$-position of the focus and the film surface at $Z=+500 \mathrm{~nm}$, $\mathrm{Z}=0 \mathrm{~nm}$, and $=-500 \mathrm{~nm}$ (Reprinted with permission from Ref. [110] @ The Optical Society). g Line plots of the surface deformations and schematics of the relationship between the $\mathrm{Z}$ position of the focus and the film surface at $Z=+2 \mu \mathrm{m}$ and $\mathrm{Z}=-2 \mu \mathrm{m}$. (Reprinted with permission from Ref [111]. ( $)$ The Optical Society)

polymer migrates in the direction of the polarization of the light from high to low light intensity regions. Effectively, the resultant polymer deformation is strongly dependent on many parameters. Ishitobi et al. discussed the effect of the incident light polarization and the position of the laser focus on the deformation pattern [110]. In particular, they found that the deformation pattern was strongly dependent on the z-position of the focused laser spot. When the z-position was exactly on the film surface the polymer moved along the polarization direction from the center to the outside of the focused spot, thus producing two side lobes along the polarization direction and a pit at the center (Fig. 3f). If the z-position was above the film surface in air, the polymer formed a protrusion coming out towards the center of the laser focus (Fig. 3f). The polymer movement was blocked by the substrate and so the protrusion was not formed, when the laser was focused into the glass substrate (Fig. 3f) [110]. 
Recently, different kinds of sources have been used to induce an azopolymer mass migration. Ishitobi et al. presented the first report of two-photon induced plastic surface deformation in azopolymer films [111]. In their work, they reported on the deformation induced by a gradient of light intensity depended by the wavelength, polarization direction of the incident laser light and z-position of the focused spot with respect to the plane of the polymer film. In this last case, divergent results respect one-photon absorption have been reported [111]. When the $\mathrm{z}$ position of the focus was below the film surface, the polymer always moved from the outside to the center of the focused laser spot along the direction of the polarization of the excitation light and formed a protrusion at the center and two dips, one at each side of the protrusion along the polarization direction (Fig. $3 \mathrm{~g}$ ). However, no deformation was induced due to the intensity of the light spot at the film being not large enough to induce two photon isomerization and polymer movement, when the distance between the focus and sample surface was larger than $5 \mu \mathrm{m}$ [111].

The use of a confocal microscope to impress photopattern on the surface of azomaterial has been widely examined $[112,113]$. Ambrosio et al. realized different topographies i.e. trenches and bumps by changing the laser scanning parameters, in particular the mutual orientation of the light polarization direction with respect to the sample scanning direction. Precisely, they observed the formation of grooves when moving the sample perpendicularly to the light polarization direction, while they obtained ridges when the sample was moved along the polarization direction [113]. In Netti's groups, photopattern were embossed on the surface of poly(disperse red 1 methacrylate) (pDR1M) polymer by confocal microscope in order to realize novel platforms for cellular investigation [33, 35, 114, 115]. Localized light was activated into specific region of interest (ROIs), in which azobenzene photoisomerization was selectively irradiated, allowing to emboss pattern with different shape and sizes e.g. concentric squares, triangles, sphere-like geometries by simply changing the geometry of the irradiated regions (Fig. 4a). Always in Netti's group, Rossano et. al employed this versatile method to emboss circular patterns on azo polymeric scaffolds for regulating fibroblast shape and mechanics (Fig. 4b) [35], while De Martino et al. used it to imprint nanopatterns on pDR1M to obtain dynamic photo-switchable platforms capable of controlling cell fate. More specifically, switching the topographical signals at a specific cell culturing time, they were able to induce a change in the cytoskeleton organization and to reverte the stem cells final commitment (Fig. 4c) [115].

\subsubsection{Randomly-distributed surface reliefs by one-beam irradiation}

The surface deformation of azopolymer films can be induced also by the irradiation of a single collimated beam under uniform light intensity and polarization distributions [116-132]. The periodic textures obtained over the illuminated area are not generated by the application of an interference pattern, thus some self-organization mechanism is involved. Leblond et al. tried to explain this spontaneous process by Fick's Law diffusion model as a phenomenological description of molecular motion [121]. Since these periodic modulations present structural analogies with the large-scale sinusoidal deformations of SRGs, they are typically referred to as spontaneous surface relief gratings (SSRG). Hubert et al. reported for the first time a spontaneous self-organization 
A

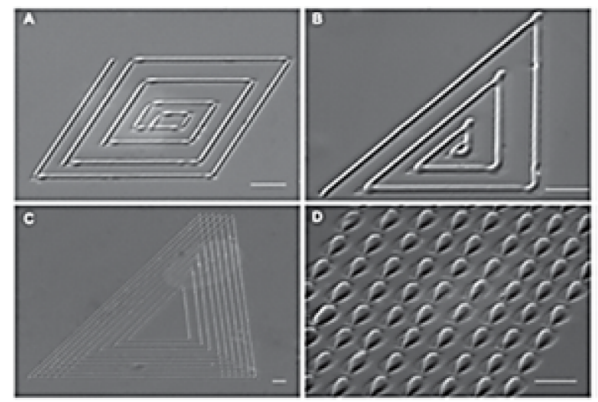

B

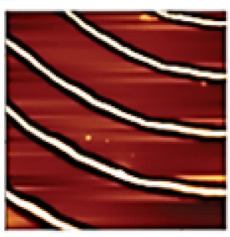

C

(1)

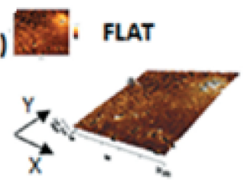

(2) IIIIII] LINEAR

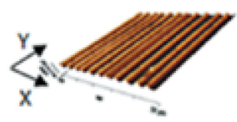

(3)

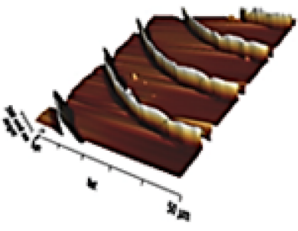

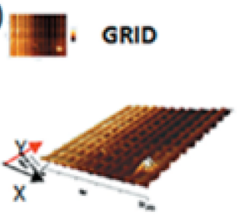
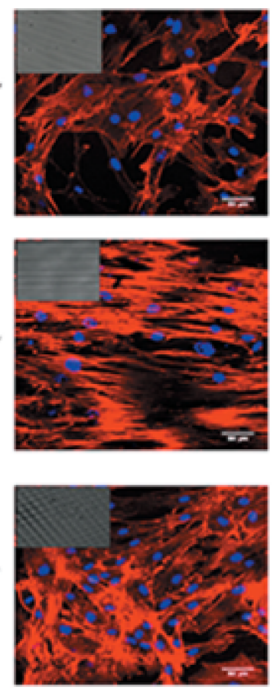

Fig. 4 a SEM images of several patterns realized on pDR1m films by using a confocal microscope, drawing several ROIs and irradiating the sample with $514 \mathrm{~nm}$ Argon laser wavelength on obtaining concentric squares, triangles, and sphere-like geometries realized by simply changing the features of the irradiated regions. (Adapted with permission from Ref. [112], (C) 2016 WILEY-VCH Verlag GmbH \& Co. KGaA, Weinheim). b Circular patterns inscribed on pDR $1 \mathrm{~m}$ with the laser confocal method. AFM images of patterned surfaces of the azo films irradiated with an Argon laser at $514 \mathrm{~nm}$ for $58 \mathrm{~s}$ (Adapted with permission from Ref. [35], (C) 2018 WILEY-VCH Verlag GmbH \& Co. KGaA, Weinheim). c (left side) AFM images of (1) Flat surface, (2) linear pattern realized by illuminating the sample with Argon laser at $514 \mathrm{~nm}$ for $42 \mathrm{~s}$ and (3) grid pattern obtained by two-steps illumination, overlapping the same initially linear pattern subsequently rotated of $90^{\circ}$. (Right side) Confocal images of hMSCs staining the cytoskeleton with Phalloidin (red) and the nucleus with DAPI (blue) on respectively flat, linear and grid surfaces (Adapted with permission from Ref. [115], (c) 2020 WILEY-VCH Verlag GmbH \& Co. KGaA, Weinheim)

of the flat surface of a thin azo film into ordered quasi hexagonal bumps by a one-beam irradiation (Fig. 5a) [133]. In the case of linear vertical or horizontal polarization of the incident laser, each elementary hexagon is observed to elongate in the same direction of the light polarization. In contrast, circular polarization or non-polarized light does not lead to any preferential direction of the structures (Fig. 5a) [133]. Furthermore, by tuning the illumination parameters, different pattern morphologies (parallel stripes, squared or turbulent structures) can be generated in azobenzene-based materials. For example, linearly polarized beams produce unidimensional periodic grooves, whose orientation depends on the light polarization direction. It is shown that the periodicity of the structure obtained with the one-beam approach can be controlled by changing the thickness of the polymer layer and the inscription temperature, in addition to the wavelength of the inscribing light (Fig. 5b) [132]. Several attempts have been carried out to understand the underlying physics that controls these various forms of pattern formation. The dependence of photoactivated pattern formation on both the light's intensity and polarization has been extensively investigated, but its physical explanation is still incomplete. Galinski and co-workers, for examples, explained the 


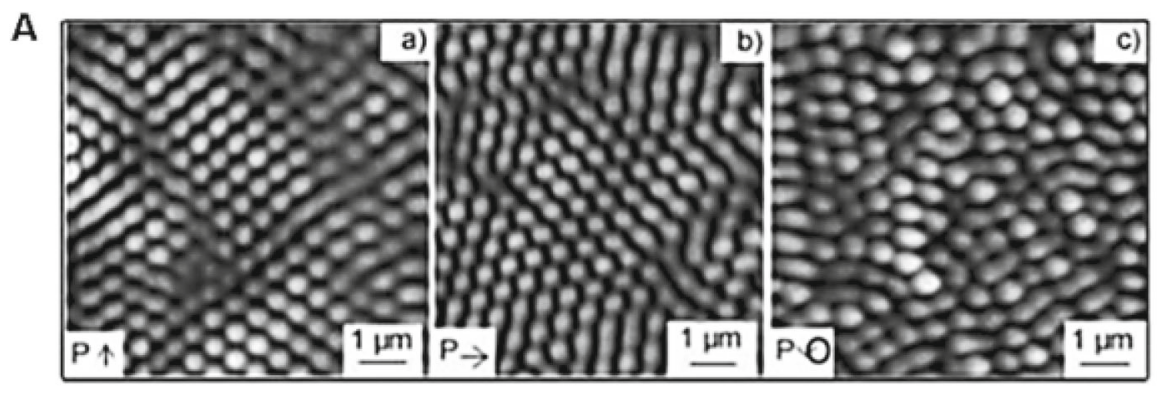

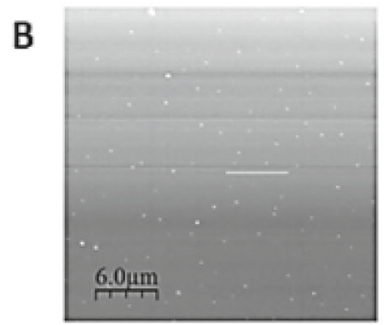

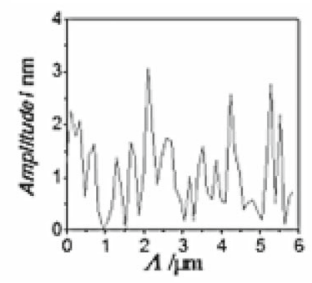

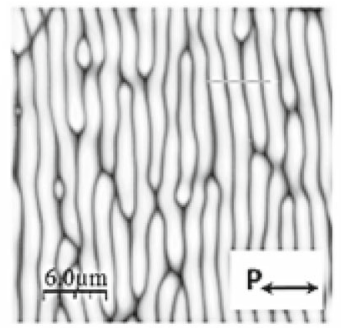
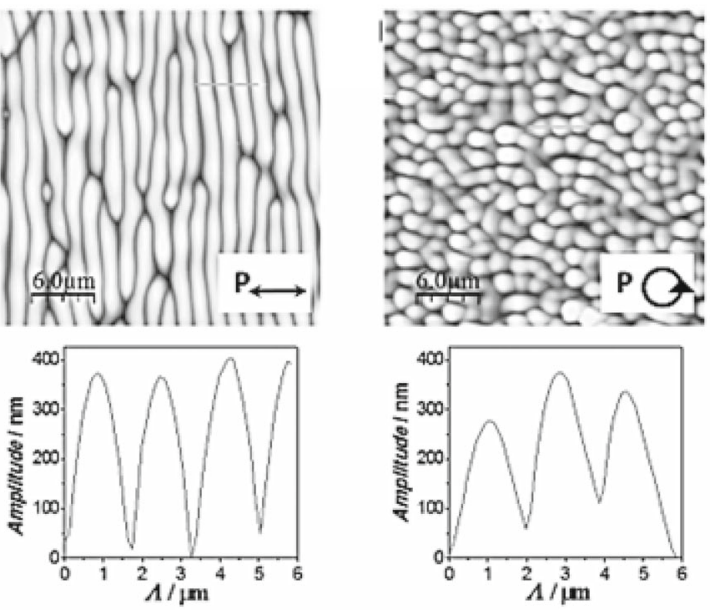

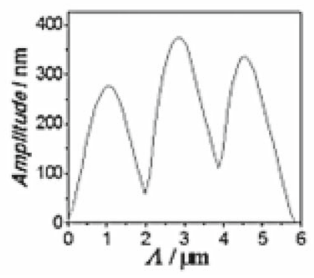

Fig. 5 a A.F.M. images of hexagonal structures for different incident laser polarisations: vertical, horizontal, circular (Reproduced with permission from Ref. [133], (c) (2003) COPYRIGHT Society of Photo-Optical Instrumentation Engineers (SPIE). b The atomic force microscopy scans with profiles of the surface topography of a thin film of $\mathrm{P} 4 \mathrm{VP}(\mathrm{OH}-\mathrm{DMA})$ prior to irradiation and after irradiation with a linearly polarized and a circularly polarized (Reproduced with permission from Ref. [132], (C) 2016 WILEY-VCH Verlag $\mathrm{GmbH} \& \mathrm{Co}$. KGaA, Weinheim)

pattern formation entirely based on principle of phase separation in the polymer [128]. Using experiments and simulations, they showed that phase separation is enhanced by polarized light on azopolymer and it is caused by an instability created by the photoactivated transitions between two immiscible states of the polymer. These two phases tend to separate leading to a spatial reorganization of the system [128].

\subsection{Directional photofluidization lithography (DPL)}

If azomaterials properties are coupled with other fabrication techniques, micro/nano structures of different shape and size can be realized [11, 134, 135] e.g. parallel groves $[32,107,112,136]$, array of microporous [137-141] and hemispherical caps [142, 143], whose geometry can be successively modified. Indeed, this technique allows to produce very sophisticated micro/nanoarchitecture that would be difficult to attain with other methods $[12,107,144,145]$. The morphology of these prefabricated structures 
can be reshaped by a light irradiation step according to the general polarizationdependent directionality of the mass migration phenomenon. This process, known as directional photofluidization lithography (DPL), allows for the reconfiguration of the initial 3D structure after the irradiation of a linearly polarized light, where azopolymeric structures became fluidized at room temperature, and their directional flow follows the direction of the light polarization [146]. It is a light-induced athermal fluidization of azobenzene molecule-containing materials under light irradiation. The term "photofluidization" indicates the photo-softening of azomaterials by repeated photoisomerization of azobenzene molecules. The advantages of using pre-structured architectures on azopolymer surfaces include the feasibility of the optical setup for light irradiation with respect to the conventional photolithography, the possibility to induce a uniform photo-fluidization over a large-area under illumination and the tunability of the geometry of the superficial pattern, which can be changed several times $[9,146]$. Eventually, 3D complex structures, not easily producible by traditional methods, can be obtained e.g., domes, mushrooms, lemons, canoes from a regular array [144]. There are several examples in literature of azopolymer patterns which are subsequently modified by light irradiation [107, 145]. Lee et al. fabricated a linear azopolymer array by soft lithography and successively photo-reconfigured it by holographic photofluidization, tuning the polarization of the interference patterns [136] (Fig. 6a). They reported also the formation of ellipsoidal hole array as a consequence of the reconfiguration of a line array upon irradiation of two interfering beams perpendicularly to the array $[107,136]$. Moreover, Lee and co-workers produced for the first time a set of pillars containing-azobenzene using a PDMS negative master [144]. However, an excessive photofluidization could result in a material flow outside the initial volume impairing the reversibility of shape modification. In order to avoid this phenomenon, Pirani et al. increased the mechanical response to light of pillars using a polymer blend, polymethylmethacrylate (PMMA) within a PAZO-PMMA mixture. This blend formulation allows a reversible and controlled deformation of the micro-pillars by periodically tuning the laser polarization in time reducing also the degradation of the structure morphology over several cycles (Fig. 6b) [13]. Additionally, if an array of cylindrical azopolymer micro-posts is irradiated with circularly polarized light, it reshapes in an array of 3D microstructures having a re-entrant geometry similar to a mushroom (Fig. 6c), showing a strong repellence to the wetting of almost any liquid [14]. Conversely, irradiation of a cylindrical micropillar array by linearly polarized results in the asymmetric structures with wetting anisotropies along different directions of the film $[11,13,147]$.

As mentioned previously, the initial pillar array is usually fabricated by soft lithography. A more innovative approach uses the photo fluidization properties of azobenzene to fabricate nanostructures and not only for modifying already existing arrays. Choi et al. reported the possibility to obtain directly patterned superficies from micrometer to sub-100 $\mathrm{nm}$ range by vertical-directional photofluidization of azobenzene materials [10]. They showed the advantage of obtaining structures into a range which is typically not feasible by soft lithography technique. In particular, they focused on vertical directional movement of azobenzene materials instead of, largely investigated, lateral directional movement of azobenzene materials. To do this, the azobenzene materials film was put in contact with patterned elastomeric molds (i.e., PDMS). Suc- 
A
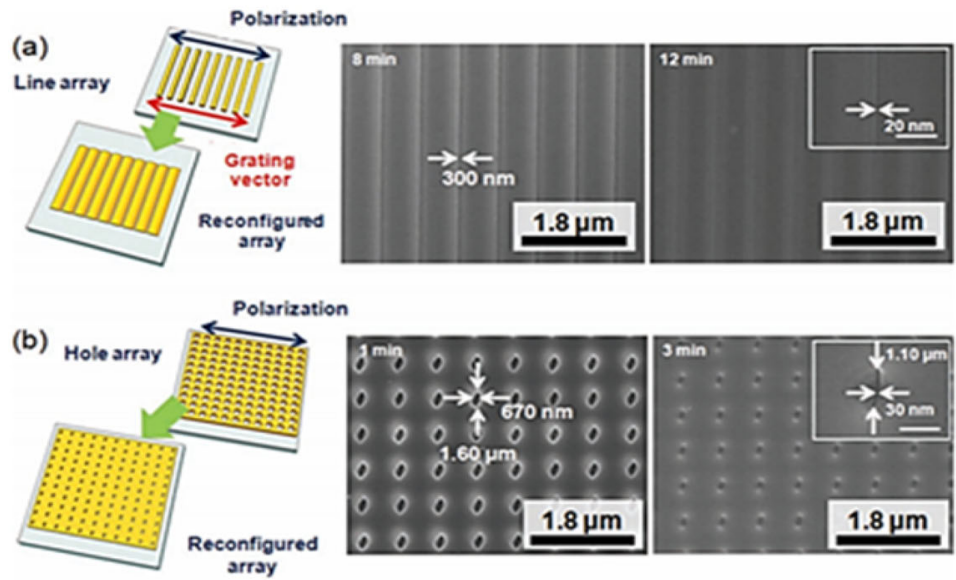

B

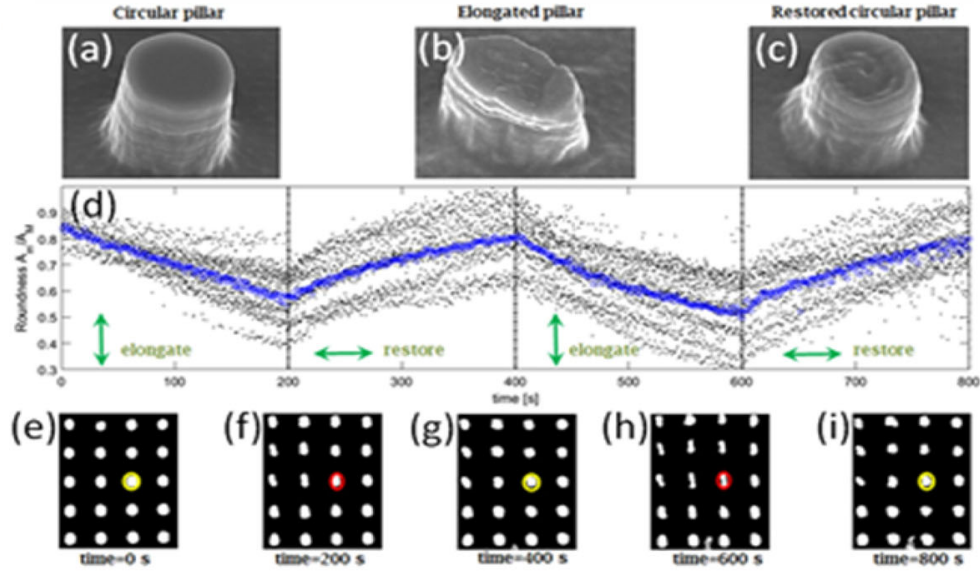

C

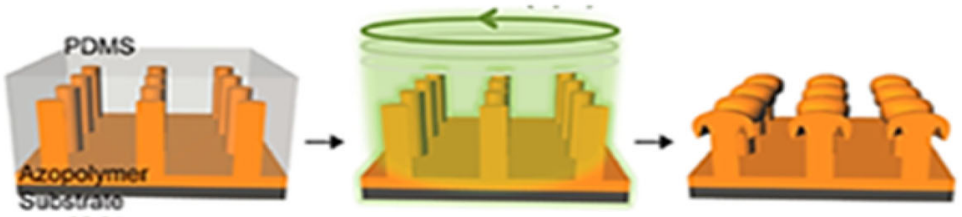

Fig. 6 a Schematic diagram of directional photo fluidization (right) and SEM images of photo-reconfigured arrays of different shapes upon linearly polarized light irradiation (left) (Reproduced with permission from Ref. [136], Copyright ( 2011 WILEY-VCH Verlag GmbH \& Co. KGaA, Weinheim). b SEM pictures of individual pillars: before light irradiation, after a single laser exposure with linear polarization (elongated, elliptical cross section) and after two laser exposures with two orthogonal polarizations (restored circular cross section) (upper part). Time-resolved roundness values for pillars during laser irradiation with timevarying polarization states (black dots: roundness of individual pillars, blue circles: mean value over pillars in each frame) and binarized optical images of micro-pillars at relevant times during pillar light-modification (lower part) (Reproduced with permission from Ref. [13], Copyright @ 2016, Springer Nature). c Schematic illustration showing fabrication procedures for the mushroom-shaped micropillar array: soft-molding of azopolymer to form the micropillar array and illumination with circularly polarized light to reconfigure the micropillars to have mushroom-like heads (Adapted with permission from ACS Nano 2017, 11, 8, 7821-7828. Copyright () 2017 American Chemical Society [14]) 
cessively, submicrometric structures were fabricated by two-step illumination, first a slantwise circularly polarized light was directed onto azomaterial film in contact with the PDMS, followed by a perpendicular irradiation. Under illumination, the polymer drifted upward along the cavities of the molds resulting in pattern formation. This light-induced fabrication was defined directional photofluidization imprint lithography (DPIL) and provided also a facile way to fabricate complex hierarchical multiscale structures [10].

\subsection{Stimuli-responsive LCEs containing azobenzene}

Photo-induced motions in azobenzene-based systems range from chromophore reorientations to material displacement, resulting in anisotropic optical properties e.g., birefringence, surface patterning, or even in macroscopic shape changes [2]. Similarly, we can attribute each of these phenomena to different classes of materials. Above, we have introduced the photo-induced mass transport that occurs in amorphous materials, while here, we focus on reorientational chromophore motions at the domain level that take place in liquid crystalline or semicrystalline materials. Azobenzenes are robust and anisotropic molecules that with suitable substitutions can show liquid crystalline behavior [65]. The azo chromophores act as mesogens, and their photoalignment becomes transferred to the LC host [65]. A very small azo content can lead to orientational control of LC domains [148] so that the azo molecular motion is amplificated in LC systems. The combination of LCs (order) and polymeric materials leads to liquid-crystalline polymers (LCPs) characterized by high-performance and typically uncrosslinked macromolecules. When LCPs contain densely crosslinked architecture, they are commonly referred as liquid-crystalline networks (LCNs). Furthermore, liquid-crystalline elastomers (LCEs) are LCNs with flexible polymer backbones, typically polysiloxane, and low crosslink density. In particular, the weak crosslinking provide them with fascinating mechano-optical properties, making them promising materials for artificial muscles [61, 149]. Moreover, liquid-crystal elastomers (LCEs) are characterized by an ordered organization as normal LCs, in which the molecular constituents of the material are preferentially aligned in a liquid crystalline phase. Depending on the mode of alignment of mesogens in LCEs, they are classificated as nematic LCEs, smectic LCEs, cholesteric LCEs [149]. In addition, the design of an LCE architecture involves the inclusion of slightly dense crosslinks, which confer specific structural and elastic properties to the polymeric network. The azobenzene moiety typically acts both as a cross-linker and deformation trigger molecule [150]. In order to obtain photo deformable cross linked LCPs, high-quality systems are obtained when azobenzene moiety is covalently bound to the polymer networks. Accordingly, LCPs can be cross-linked by either non-mesogenic or mesogenic molecules and form moderately to densely cross-linked network architectures (LCNs) or lightly cross-linked elastomers with flexible backbones (LCEs) [151]. By incorporating azobenzene into LCEs, it is observed a contraction of LCE film upon exposure to UV light to cause a photo-isomerization of the photochromic moiety. It can induce a reduction in the nematic order and causes a contraction of the films upon exposure to UV light as a result of a photoisomerization which therefore can induce a phase transformation 
from the nematic to the isotropic state [152-154]. In fact, in an azobenzene-containing LCN, photochemical LC-I phase transition will make the polymer backbone lose its anisotropic nature and adopt into an isotropic chain conformation, resulting in the whole system changing its shape (Fig. 7a) [155, 190]. This is due to the UV-induced cis trans isomerisation of the azo- $(\mathrm{N}=\mathrm{N})$ bond, whereby the photo-sensitive mesogenic molecules change from a rod-like shape that stabilise the liquid crystal phase, to a kinked shape that destabilising the nematic phase, reducing its order parameter. The principle consequence of such photo-isomerisation is the destabilisation of the nematic phase, whose order parameter depends on temperature in a near-critical fashion [156]. Ikeda et al. reported the first example of a photochemical phase transition in LC polymers doped with low-molecular-weight azobenzene molecules due to azobenzene isomerization that induce a nematic-isotropic phase transition [148, 157]. The phase separation is observed in doped systems when the concentration of the azo molecules is high, thus LC copolymers appeared on the scene as better candidates [153]. Among the earliest examples, such mechanical photo-induced actuation in nematic elastomers was reported by Finkelmann et al. [158]. They reported a pioneering work on large contractions of LCNs containing azobenzene moieties by light [158]. Monodomain nematic LCEs containing polysiloxane main chain and azobenzene chromophores at the crosslinkers, showed a contraction of $20 \%$ upon exposure to UV [158]. Moreover, Keller et al. synthesized monodomain nematic azobenzene sideon elastomers that showed fast photochemical contraction up to $18 \%$ by UV irradiation [159]. Other than one-dimensional and two-dimensional (contraction or expansion), also three-dimensional movements (bending and unbending) of LCE film have been demonstrated. Such bending behavior is determined by the large absorption extinction coefficient of the trans-azo molecule in the UV optical range, which generates a gradient in the photoisomerization efficiency for the azo molecules located at different positions along the direction of the film thickness. Indeed, most of the incident light is absorbed in the proximity of the irradiated surface, while the trans-azo molecules in the bulk of the film and at the bottom surface remain almost unaffected [9, 154]. This isomerization gradient causes the contraction only of the exposed surface of the film, which finally bends toward the incoming light direction. Ikeda et al. reported an anisotropic bending and unbending induced by light of cross-linked liquid crystalline polymer films containing an azo-benzene moiety and a diacrylate-azobenzene moiety. The absorption of photons occurs only in the surface of the film due to the high absorption coefficient of azobenzene so the volume contraction is induced only in the surface region of the cross-linked LC polymer film upon irradiation [160]. Furthermore, Yin et al. proposed an interesting crosslinked liquid crystalline polymers containing azotolane moieties in side chains which show a photoinduced bending and unbending behavior upon irradiation with sunlight, according to the trans-cis photoisomerization of the azotolane moieties [161]. LCPs can be classificated from the alignment of the mesogens as monodomain or polydomain. Monodomains LCPs feature global alignment and show large anisotropy in various macroscopic properties, while in polydomain LCPs, the mesogens align in the same direction only in local domains and the direction of each domain is random [162]. They prepared monodomain and polydomain polyacrylate films containing azobenzene moieties in the side chains and crosslinks. When the monodomain film was irradiated with UV light, 
the film bent toward an actinic light source, while it returned to the initial flat state when irradiated with visible light $(>540 \mathrm{~nm}$ ). The bending and unbending behaviour was reversible just by changing the wavelengths of the incident light. Furthermore, they observed that the bending can be anisotropically induced only along the rubbing direction of the alignment layers. In fact, also if the film was rotated by $90^{\circ}$, the bending is always observed along the rubbing direction. Polydomain films showed more fascinating behaviour when irradiated with linearly polarized light. The bending axis could be tuned by the polarization state of the light. This selective direction bending cannot be obtained through thermal processes [162]. The bending extension of the film depends on the cross-linking density, higher density holds a higher order parameter and a larger volume contraction along the rubbing direction [163]. Furthermore, the bending angle differs with different azo concentration. In a system with high azobenzene concentration, photons are absorbed by a very thin surface layer; high crosslinking density enhances the interaction between the surface layer and the bottom layer, thus making it easier for the surface layer to drive the bottom layer, and causing larger deformation. On the contrary, in a system containing a small amount of azobenzenes, light can penetrate deep enough in the sample, so the bending angle is dominated by the modulus of the film. Larger modulus generated from higher cross-linking density makes it hard to bend the film, leading to decrease of the bending angle [155]. For example, Yu et al. prepared a series of monodomain crosskinked LCP films with different cross-linking densities using monomers all containing azobenzene [163]. It was observed that the films with different cross-linking densities exhibited different bending extents and speeds. Furthermore the birefringence of the films measured at room temperature was found to increase with the increment of the cross-linking density due to the effect of the different order parameters in the films [163]. Ikeda et al. showed that a single film of a liquid-crystal network containing an azobenzene chromophore can be repeatedly and precisely bent along any chosen direction by using linearly polarized light. A reversible and directional photo-selective film bending is achieved only in the direction of the linearly polarized light due to the orientation-dependent efficiency of the light absorption for the trans-azo molecules aligned with the light polarization direction that drives an efficient order-disorder transition (Fig. 7b) [160, 190]. This striking photomechanical effect results from a photoselective volume contraction and may be useful in the development of highspeed actuators for microscale or nanoscale applications, for example in microrobots in medicine or optical micro tweezers [164-169]. Spacer length of LC monomers proved to play an important role in determining the mesomorphic properties of cross-linked LCPs, so it will influence their deformation. Azobenzene-containing cross-linked LCP films with the long spacer of undecylene with different crosslinking densities demonstrated different bending behavior. When LCP film with the low crosslinking density was exposed to UV light and heated above $90{ }^{\circ} \mathrm{C}$, the film bent directly towards the irradiation direction of the actinic light, whereas the bending towards the irradiation direction was preceded by the bending away from the light source when the temperature was below $90{ }^{\circ} \mathrm{C}$. This distinct bending phenomenon was not observed in the CLCP films with higher crosslinking densities, all of which bent directly towards the light source (Fig. 7c) [170]. Over that bending, others three-dimensional movement such as oscillating, twisting and swimming of LCE films containing azobenzene can 

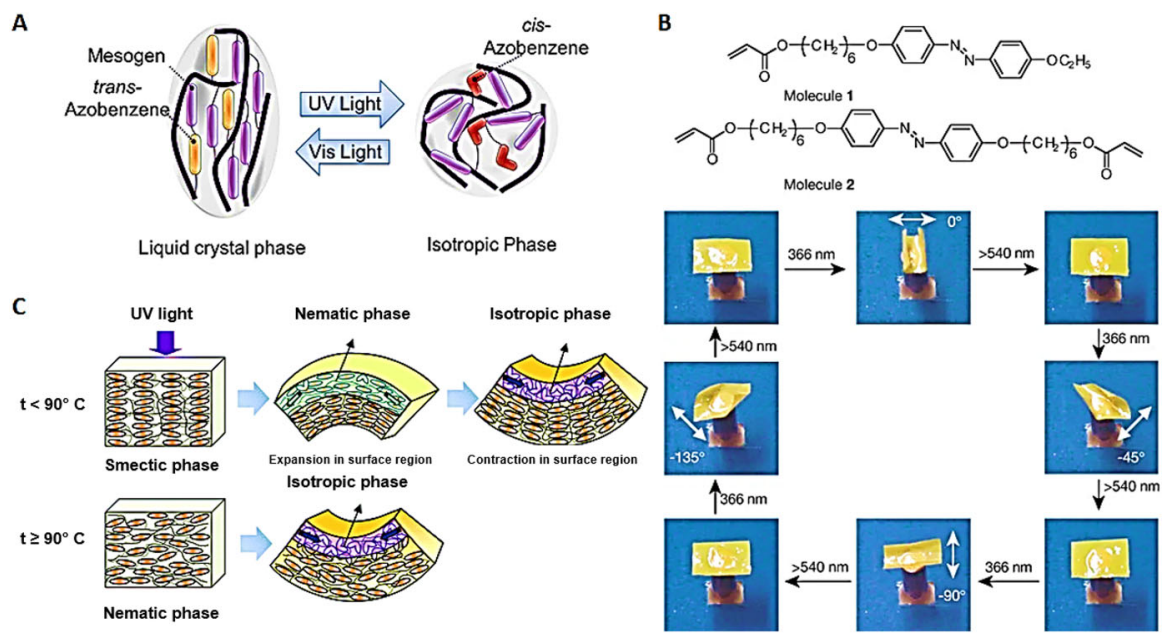

Fig. 7 a Photoinduced deformations of cross-linked liquid crystal polymers based on photochemical phase transition (Reproduced with permission of Chinese Physical Society from Ref. [155]). b Photographs of the polydomain film in response to irradiation by linearly polarised light at different angles of polarisation (white arrows) at $\lambda=366 \mathrm{~nm}$; the bent films are flatted by irradiation with visible light at $\lambda>540 \mathrm{~nm}$ (Reproduced with permission from Ref. [160] copyright (C) 2003, Nature Publishing Group). c A schematic illustration of a plausible mechanism of the different bending modes of F9010 at different temperatures (Republished with permission of Royal Society of Chemistry from Ref. [170]; permission conveyed through Copyright Clearance Center, Inc.)

be observed by irradiation with light [171]. Broer et al. fabricated LCE film with diacrylate dopants containing azobenzene moieties blended with liquid crystalline diacrylate hosts and photopolymerized in a twisted configuration. Exposure to UV radiation induced anisotropic expansion/contraction, and simple variations in geometry were used to generate uniaxial bending or helical coiling deformation modes [172]. Systems with a splayed or twisted nematic (TN) director profile drive greater amplitude and faster bending than uniaxial planar systems with the same chemical composition [173]. Furthermore, by varying the orientation of the molecular director through the thickness of the film actuator can be obtained large performance in light-driven liquid-crystal network (LCN) actuators [173]. Similarly, Fletcher and Katsonis et al. reported a similar complex motion of twisted aligned LCN materials made by adding chiral dopants into nematic LCNs. The films had different geometry according to the cutting direction, in which the orientation of the liquid-crystal direction changes $90^{\circ}$ from the bottom surface to the top surface. Under irradiation with UV light, the ribbons displayed different motion, such as winding, unwinding, and helix inversion [174]. Ikeda and Shishido et al. proved that the location of azobenzene moieties also affected the films' bending behaviours. They prepared two azobenzene-containing LCN films with the same cross-linking density and azobenzene concentration, but with different binding positions of azobenzenes to the polymer network. Under similar irradiation conditions, a sample bearing the azobenzenes as cross-links bends toward the light source, whereas a sample containing the azobenzenes as side chains bends away from it [175]. 


\subsubsection{Light-induced permanent surface topographies in azo cross-linked network coatings}

LCNs are created from reactive LC monomeric mixtures that can be aligned by a variety of techniques. Once achieving the desired alignment, the monomeric mixture can be photopolymerized leading to cross-linked liquid crystal polymer networks. The ability to design and fabricate the anisotropic coating to respond to specific commands leads to the creation of many different directed topographies. Most interestingly, UV exposure of prealigned azo-containing LCN coatings induces the creation of topographies without the need of optical set-up [150]. Liu et al. induced changes in the topology of the coating surface of a cross-linked polymer network i.e., $p$-methoxyphenol modified chiral-nematic film by means of light. The photoinduced isomerization of an integrated azobenzene compound initiates a change of the order of the liquid crystal molecular units in the network. By exposing the film locally by a line mask, the volume increase forms a protrusion at the exposed area. The deformation can only be observed under the condition of continuous irradiation with UV light, and while it recovers its initial state and the structures disappear when the UV light is switched off. While, when the polymer networks have been formed in the presence of a chain transfer agent, the protrusions that are formed remain after removal of the light source (Fig. 8a) [176]. Furthermore, they observed also that if the surface deformation is predominantly photoinduced, there is also a thermal contribution due to local heating of the sample by the absorption of light. Instead, when using a coating polymerized in the isotropic phase, there is nearly no expression of topographies upon selective UV exposure. The topography formed in the isotropic cross-linked coating results solely from thermal effects. Interestingly, when the azobenzene is swapped for a simple UV absorber such as Tinuvin 328, with an absorption spectrum that coincide with that of azobenzene but with short geometrical changes upon UV absorption, the topographies formed by selective illumination are lower in height than those generated using the azobenzene moiety (Fig. 8a) [176]. Stable topographies require the presence of scavengers during polymerization of the LC coating. Reversible surface topographies in contrast with the permanent surface topographies, are prepared in the absence of any radical scavenger [150]. We have largery mentioned the azobenzene-containing linear polymers that form surface reliefs based on light-driven material transport. Liu et al. also use light for the dynamic actuation of a surface relief. They advance obtained photo-switchable surface topologies through integration of azobenzene-containing crosslinkers into a structured chiral nematic network. The liquid-crystal network contained striped patterns of alternating areas with chiral nematic order and homeotropic orientation. With this alignment setup, the coating contains areas that expand and contract in thickness adjacent to one another, resulting in larger differential topographical features (Fig. 8b, c) $[177]$.

\section{Dynamic azopolymer surfaces in cell biology}

The necessity to control and guide cell functions has led to the development of dynamic bio-interfaces for applications in tissue engineering, tissue repair and cell biology 

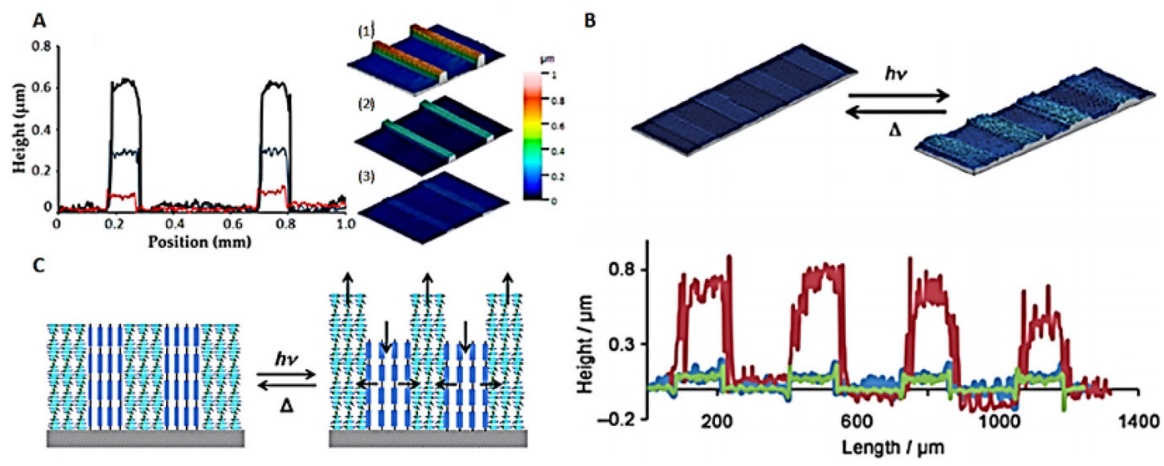

Fig. 8 a Surface profiles in cross-section and in 3D view of protrusions made by exposing the $p$ methoxyphenol modified chiral-nematic film through a line mask with a periodic pitch of $500 \mu \mathrm{m}$ and an opening of $100 \mu \mathrm{m}$. The pictures were made by interference microscopy and show the profiles of the chiral nematic film with azobenzene (black line in (1)), Tinuvin (blue line and (2)), and the film with azobenzene polymerized in the isotropic state (red line and (3)) (Adapted with permission from Macromolecules 2012, 45, 19, 8005-8012. Copyright (C) 2012 American Chemical Society [176]). b Upper part, 3D images of surface topologies at the original state (left) and during illumination with UV light (right). Lower part, surface profiles of the initial profile (blue), during UV illumination (red), and after removal of the illumination (green) (Reproduced with permission from Ref. [177], Copyright @ 2012 WILEY-VCH Verlag GmbH \& Co. KGaA, Weinheim). c Liquid-crystal network containing striped patterns of alternating areas with chiral nematic order and homeotropic orientation (Reproduced with permission from Ref. [177], Copyright @ 2012 WILEY-VCH Verlag GmbH \& Co. KGaA, Weinheim)

[178]. These smart surfaces are engineered in order to provide in space and in time precise instructive cues to cells, thus enhancing specific biological events. Indeed, biochemical and biophysical signals are known to affect cellular functions such as adhesion spreading, migration, proliferation and differentiation [179]. The transmission of physical cues to cells occurs upon cell-material interactions at the interface, e.g. through a communication between the external environment and the inner cytoskeleton mediated by cells membrane receptors. In addition, the native extracellular environment (ECM) has been demonstrated to be a dynamic entity, continuously undergoing remodeling, assembly and degradation processes during growth and in disease progression [180]. Thus, materials with tunable properties have obtained great attention for providing dynamic and reversible cell culture platforms recapitulating the original cellular microenvironment. Azobenzene-type materials are commonly employed for the development of dynamic bio-interfaces because of their ability to change the shape of surface topography in response to light. This property allows to create interfaces, whose properties can be reversibly modulated on-demand through the application of a light field. Moreover, light-controlled systems are mostly preferred in biological applications because, among all external stimuli used to trigger responsive materials, light provides high spatial/temporal controllability and is not dangerous for cells, thus giving the possibility for in-situ studies of cellular behavior. In addition, the formation of surface protrusions on azopolymer materials upon light irradiation provides a physical nano/micro pattern, which can be exploited as guidance structures to orient and align cells along preferential directions. 


\subsection{Recent advances in azopolymer patterning for cell culture}

Baac et al. were the first to use SRGs on submicron-scale produced on the surface of azobenzene material by holographic light in order to control cell orientation and alignment. They cultured human astrocytes (HA) on the patterned surface, which after a few days of proliferation appeared to be oriented and elongated along the SRG direction.[24] Azopolymer patterns appeared soon suitable systems to direct cell adhesion and orientation, as well as platforms suited to create dynamic interfaces that can impart specific biomechanical cues to cells with a high space and time control. Thus, Rianna et al. investigated NIH-3T3 murine fibroblasts response to reversible SRGs imprinted and erased on a glassy azopolymer surface by an interference pattern projected by a Lloyd's mirror configuration and an incoherent light, respectively (Fig. 9a) [32]. Also Barillé et al. investigated the writing and erasure effect in presence of cells immersed in a buffer solution of phosphate buffered saline (PBS), reporting the formation of irregularities in the grating pattern due to the diffusion and divergence of the light propagating in the PBS solution [25]. In more details, they examined PC12 cells orientation and alignment on stripe-patterned (groove/ridge patterns) bio polymer surfaces generated either by the projection of an interference pattern or by the molecular self-organization as a result of one-single laser beam illumination. Furthermore, the implementation of a confocal set-up to emboss photo-patterns on azopolymeric materials with different shapes has revealed to provide high versatility and the possibility to observe cell response in real-time. In fact, Rianna et al. performed topographic changes in-situ presenting dynamic signals to cells by "erasing" and "re-writing" the imprinted pattern with a different geometry [33]. The same method was employed also by Rossano et al. in order to realize micrometer-scale circular patterns on azopolymer having concentric circular rings with pattern periodicity of $5 \mu \mathrm{m}, 10 \mu \mathrm{m}$, or $15 \mu \mathrm{m}$ affecting NIH-3T3 cell morphology and mechanical properties [35]. In addition, Fedele et al. exploited the versatility of this technique to study the effect of changes in topographical cues during early-stage angiogenesis through real-time photopatterning of an azobenzenecontaining polymer (pDR1m). They employed a 3D angiogenesis assay based on the use of spheroids derived from human umbilical vein endothelial cells and investigated the effect of dynamic topographic signal presentation on sprout formation, confirming the influence of the underlying photo-inscribed pattern on sprout directionality [114]. Furthermore, several works reported the possibility to affect cell differentiation by tuning dynamically topographical cues, revealing the potential to control and guide cell fate [181-183]. In particular, De Martino et al. induced topography changes actuated by light at specific times to reverse the fate of otherwise committed human mesenchymal stem cells (hMSC) toward osteoblastic lineage [115]. More specifically, they switched from linear parallel stripes patterned to flat or grid surfaces of azopolymer material (pDR $1 \mathrm{~m})$ realized by means of confocal laser beam illumination, inducing cellular and nuclear stretches, thus proving that modifications of cytoskeleton organization can affect gene expression and then cell fate.

There are also some examples of 3D azobenzene patterns subsequently illuminated by light used as dynamic cell culture platforms. Puliafito et al. presented an approach to spatio-temporally control substrate topographies starting from a pre- 

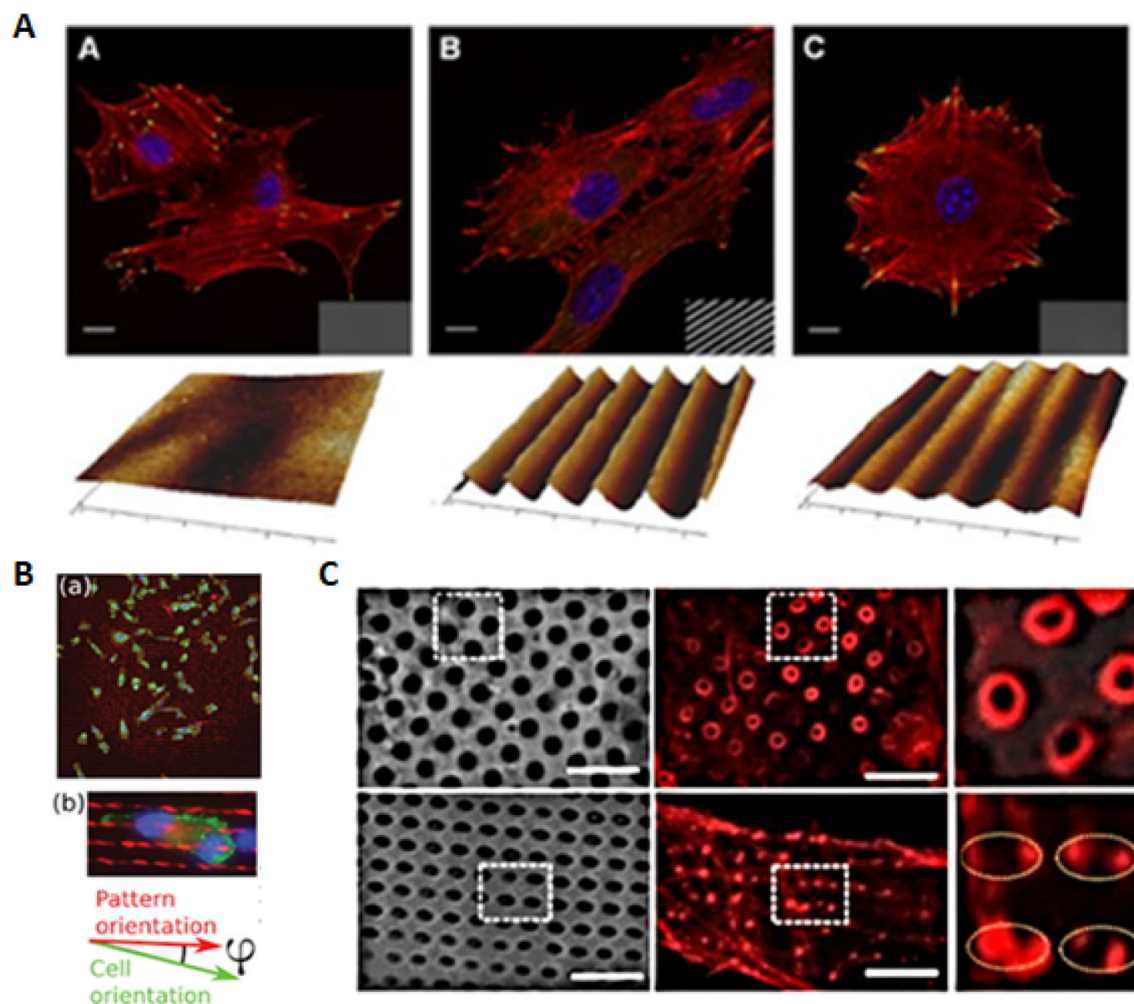

C

D
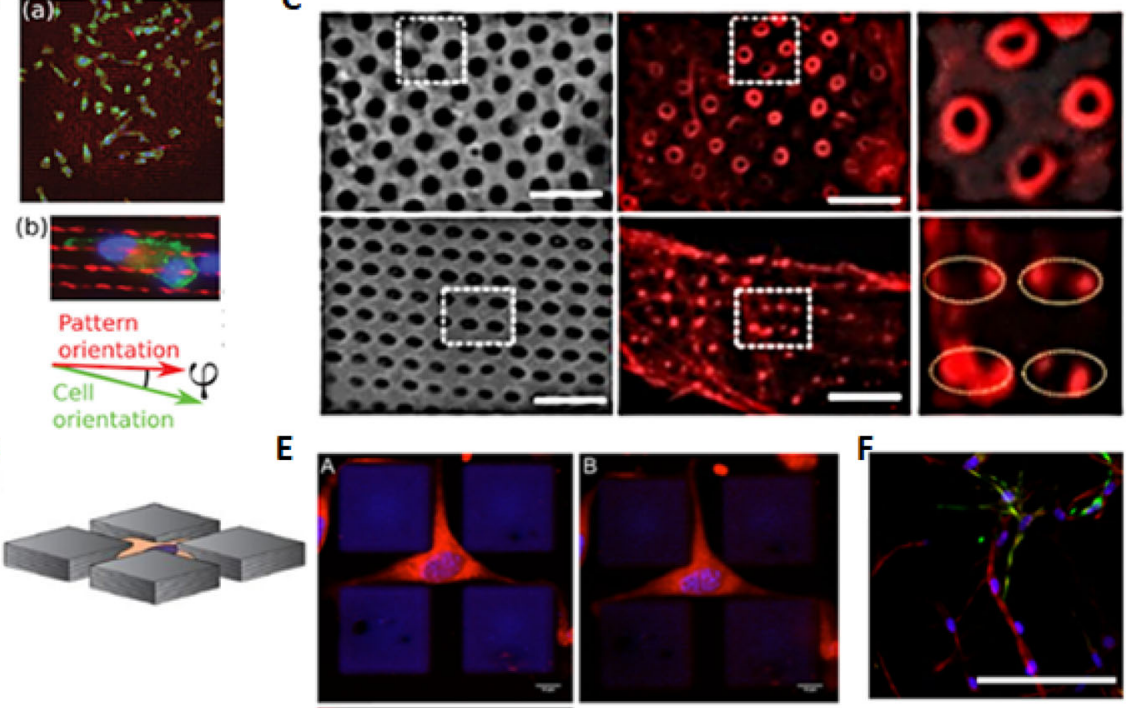

Fig. 9 a Confocal images of NIH-3T3 cells cultivated on (left) flat pDR1m substrate, (middle) SRG grating, and (right) pattern erased with circularly polarized light and plots with cell elongation and orientation (Adapted with permission from ACS Appl. Mater. Interfaces 2015, 7, 31, 16984-16991. Copyright $@ 2015$ American Chemical Society [32]). b In the upper part, images of stained cancer cells on deformed substrates. In the lower part, sketch of the conventional directions used in the figure [36]. c Brightfield and fluorescence images of U2OS cells transfected with Lifeact-RFP on azo structures, before (upper part) and after (lower part) deformation. Lifeact-RFP appears as a ring around nanopillars and preferentially accumulates to the ends of azo bars (Adapted with permission from Nano Lett. 2020, 20, 1, 577-584. Copyright $($ ) 2019 American Chemical Society [12]). d Graphical representation of the gelatin platform (Adapted with permission from ACS Appl. Mater. Interfaces 2018, 10, 1, 91-97. Copyright @ 2017 American Chemical Society [187]). e MP images of NIH-3T3 living cells before light irradiation and after 10 min of MP stimulation (Adapted with permission from ACS Appl. Mater. Interfaces 2018, 10, 1, 91-97. Copyright $(\odot)$ 2017 American Chemical Society [187]). f Immunostaining for $\alpha$ SMA (green), f-actin (red), and nuclei (blue) in $4 \mathrm{wt} \%$ PEG, 38\% non-degradable linker after 5 days of culture (Adapted with permission from Biomacromolecules 2015, 16, 3, 798-806. Copyright ( 2015 American Chemical Society [188]) 
patterned azopolymeric structure on a glass-bottom Petri dish. In detail, they modified the topography with a single exposure of the target area with living cells attached to the substrate. Specifically, depending on the local polarization orientation and intensity of the laser, micropillars get elongated along a direction parallel to the laser polarization, thus resulting in a generally both anisotropic and inhomogeneous microtextured surface. They showed that both cancer cells and kidney epithelial cells are able to sense the local deformation direction and orient and migrate accordingly (Fig. 9b) [36]. De Martino et al. also examined the possibility to dynamically manipulate cell membrane curvature by reshaping 3D azobenzene structures. In particular, they fabricate three dimensional azobenzene-based polymer structures that change from a vertical pillar to an elongated vertical bar shape upon green light illumination. They demonstrated that the dynamic pillar reshaping induced a change of local membrane curvature of U2OS cells, thus affecting actin fibers re-organization (Fig. 9c) [12]. As introduced, incorporating azobenzene into LCEs, macroscopic mechanical motions of azo systems can be observed. Further, the potentiality of these interesting motions can be employed to create dynamic light-controlled scaffolds in the biological field.

Koçer et al. designed azobenzene based LCN systems to form hybrid surfaces presenting micrometer scale topographical cues and changes in nanoscale roughness at the same time to direct cell migration [184]. A mixture of (meth)acrylate functionalized azobenzene and liquid crystalline monomers were used to create a chiral nematic phase that is subsequently aligned in plane by shear forces and then photopolymerized. Mask illumination of the films lead to local trans to cis isomerization of azobenzene molecules, resulting in a local formation of protrusions (volume generation) in the illuminated areas yielding (microscale) topographical cues for cells. This study showed that the cell speed and migration patterns were strongly dependent on the height of the (light-responsive) micrometer scale topographies and differences in surface nano-roughness. More precisely, cell motility patterns could be switched from static to highly mobile by increasing the height of the pillars or from dynamic to moderately static by increasing the nanoscale roughness or to highly static on a selective topographic pattern. Hendrikx et al. also performed preliminary studies of cells adhesion and survival on visible light responsive liquid crystalline polymer material doped with fluorinated azobenzene that reversibly changes its surface topography upon light illumination. Specifically, a mask illumination with green light of the films leads to local trans $\rightarrow$ cis isomerization of fluorinated azobenzene molecules, producing local protrusions in the illuminated areas that can be rapidly erased by blue light. NIH 3T3 fibroblast cells were cultured on the patterned surface, showing good biocompatibility, and subsequent green light illumination revealed cell shape retraction [185].

Also, with the necessity to recreate a dynamic 3D microenvironment, photoactuating hydrogels have received significant consideration. Among the photoactive groups that can be incorporated with an hydrogel matrix, the azobenzene moiety is the most known compound used to induce changes of different material properties such as the mesh size and/or its swelling behavior and shape deformations as a consequence of its photoisomerization [186]. Pennacchio et al. developed an advanced gelatin based hydrogel containing azobenzene cross-linker stimulated by light irradiation. The mixture was made of acrylamide-modified gelatin and an azobenzene-based bis-acrylamide cross-linker, which gives the photo deformable 
property to the microstructured material. In fact, they induced nuclear deformations of confined NIH-3T3 cells by light-triggered expansion of gelatin microstructures (Fig. 9d, e).[187] Rosales et al. also presented a poly(ethylene glycol)-based hydrogel with tunable stiffness due to the presence of an azobenzene-containing cross-linker, which allows to reversibly stiffening and softening the matrix upon light illumination. More in detail, upon irradiation with cytocompatible doses of $365 \mathrm{~nm}$ light, isomerization to the azobenzene cis configuration leads to a softening of the hydrogel up to $100-200 \mathrm{~Pa}$ (shear storage modulus, $G^{\prime}$ ) that is maintained over a time scale of several hours due to the long half-life of the cis isomer. The initial modulus of the gel can be recovered upon irradiation with similar doses of visible light. In these conditions, they showed high levels of survival, as well as a spread morphology of porcine aortic valvular interstitial cells trapped inside the hydrogel (Fig. 9f) [188]. Another example of hydrogel photo-actuated is reported by Lee et al., where they realized a polyacrylamide (PA)-based hydrogels with the incorporation of photoactive azobenzene cross-linker for the investigation of bone-marrow-derived mesenchymal stem cells (MSCs) morphology in response to variations of the hydrogel stiffness upon blue-light illumination [189].

\section{Conclusion}

The intense research activity on optical properties of azobenzene compounds has revealed the valuable applications of azobenzene based materials in a large variety of fields. Although photo-birefringence and optical dichroism are well-known phenomena induced by light, more effort is required to understand the complex mechanism of SRG formation, and thus mass transport, occurring on the surface of azo molecule based materials. Nevertheless, structured azopolymeric surfaces have been exploited for different purposes, involving photonic and biological applications. In this review, we have described the general aspects regarding photo-responsive properties of azomolecules embedded in amorphous polymers or LC systems, with a particular focus on the photomechanical phenomena. These phenomena are responsible for macroscopic material movement which can be directed to form specific pattern or surface protrusions. Most attention has been given to the various systems employed to generate regular or randomly-distributed surface reliefs. These include holographic light irradiation, 1D Gaussian laser beam and one-single beam illumination. Additionally, directional photofluidization lithography (DPL) has been reviewed as an emerging technique used to modify subsequently preconfigured structures, overcoming the complexities of conventional photolithography methods. In these applications, azobenzene molecules are even more frequently used as photo-active chromophores for inducing re-shaping of prefabricated structures upon light irradiation. We also described in detail the macroscopic shape deformations occurring in LC elastomers, in which phase transition is induced by azo molecule photoisomerization, highlighting the interesting outcomes as smart photo-actuators in the field of robotics in medicine and optical devices. Moreover, exciting applications of azobenzene-type materials as dynamic biointerfaces to control cell functions have been described, emphasizing the promising engagement of azopolymers as photo-responsive materials for dynamic cell culturing. 
Author contributions SDM and FM contributed equally. The manuscript was written through contributions of all authors. All authors have given approval to the final version of the manuscript.

Funding Open access funding provided by Istituto Italiano di Tecnologia within the CRUI-CARE Agreement.. Financial support was provided by IIT (Istituto Italiano di Tecnologia).

\section{Compliance with ethical standards}

Conflict of interest The authors declare no competing financial interest.

Open Access This article is licensed under a Creative Commons Attribution 4.0 International License, which permits use, sharing, adaptation, distribution and reproduction in any medium or format, as long as you give appropriate credit to the original author(s) and the source, provide a link to the Creative Commons licence, and indicate if changes were made. The images or other third party material in this article are included in the article's Creative Commons licence, unless indicated otherwise in a credit line to the material. If material is not included in the article's Creative Commons licence and your intended use is not permitted by statutory regulation or exceeds the permitted use, you will need to obtain permission directly from the copyright holder. To view a copy of this licence, visit http://creativecommons.org/licenses/by/4.0/.

\section{References}

1. P. Rochon, E. Batalla, A. Natansohn, Appl. Phys. Lett. 66, 136 (1995)

2. A. Natansohn, P. Rochon, Chem. Rev. 102, 4139 (2002)

3. C.J. Barrett, P.L. Rochon, A.L. Natansohn, J. Chem. Phys. 109, 1505 (1998)

4. J. Kumar, L. Li, X.L. Jiang, D.-Y. Kim, T.S. Lee, S. Tripathy, Appl. Phys. Lett. 72, 2096 (1998)

5. P. Lefin, C. Fiorini, J.-M. Nunzi, Pure Appl. Opt. 7, 71 (1998)

6. T.G. Pedersen, P.M. Johansen, N.C.R. Holme, P.S. Ramanujam, S. Hvilsted, Phys. Rev. Lett. 80, 89 (1998)

7. O.M. Tanchak, C.J. Barrett, Macromolecules 38, 10566 (2005)

8. K.G. Yager, O.M. Tanchak, C. Godbout, H. Fritzsche, C.J. Barrett, Macromolecules 39, 9311 (2006)

9. S.L. Oscurato, M. Salvatore, P. Maddalena, Nanophotonics 7(8), 1387-1422 (2018)

10. J. Choi, W. Cho, Y.S. Jung, H.S. Kang, H.-T. Kim, ACS Nano 11, 1320 (2017)

11. S.L. Oscurato, F. Borbone, P. Maddalena, A. Ambrosio, ACS Appl. Mater. Interfaces. 9, 30133 (2017)

12. S. De Martino, W. Zhang, L. Klausen, H.-Y. Lou, X. Li, F.S. Alfonso, S. Cavalli, P.A. Netti, F. Santoro, B. Cui, Nano Lett. 20, 577 (2020)

13. F. Pirani, A. Angelini, F. Frascella, R. Rizzo, S. Ricciardi, E. Descrovi, Sci. Rep. 6, 31702 (2016)

14. J. Choi, W. Jo, S.Y. Lee, Y.S. Jung, S.-H. Kim, H.-T. Kim, ACS Nano 11, 7821 (2017)

15. X. Pang, J.-A. Lv, C. Zhu, L. Qin, Y. Yu, Adv. Mater. 31, e1904224 (2019)

16. A. Priimagi, A. Shevchenko, J. Polym. Sci. B Polym. Phys. 52, 163 (2014)

17. Z. Zheng, Y. Lu, Q. Li, Adv. Mater. 32, 2070305 (2020)

18. G. Albano, G. Pescitelli, L. Di Bari, Chem. Rev. 120, 10145 (2020)

19. H. Rekola, A. Berdin, C. Fedele, M. Virkki, A. Priimagi, Sci. Rep. 10, 19642 (2020)

20. Y. Zhai, L. Cao, Y. Liu, X. Tan, Materials 13, 23 (2020)

21. M. Pilz da Cunha, M.G. Debije, A.P.H.J. Schenning, Chem. Soc. Rev. 49, 6568 (2020)

22. C. Zhu, Y. Lu, J. Sun, Y. Yu, Langmuir 36, 6611 (2020)

23. S. De Martino, P.A. Netti, Biophys. Rev. 1, 011302 (2020)

24. H. Baac, J.-H. Lee, J.-M. Seo, T.H. Park, H. Chung, S.-D. Lee, S.J. Kim, Mater. Sci. Eng. C 24, 209 (2004)

25. R. Barillé, R. Janik, S. Kucharski, J. Eyer, F. Letournel, Colloids Surf. B Biointerfaces 88, 63 (2011)

26. L. Rocha, C.-M. Păiuş, A. Luca-Raicu, E. Resmerita, A. Rusu, I.-A. Moleavin, M. Hamel, N. BranzaNichita, N. Hurduc, J. Photochem. Photobiol. A Chem. 291, 16 (2014)

27. M. Ventre, C.F. Natale, C. Rianna, P.A. Netti, J.R. Soc, Interface 11, 20140687 (2014) 
28. C. Rianna, M. Ventre, S. Cavalli, M. Radmacher, P.A. Netti, A.C.S. Appl, Mater. Interfaces 7, 21503 (2015)

29. R. Barillé, P. Codron, G. Mabilleau, F. Manero, R. Mallet, S. Zielinska, E. Ortyl, J. Eyer, F. Letournel, Open Biomed. Eng. J. 12, 1-15 (2018)

30. M. Salvatore, S.L. Oscurato, M. D’Albore, V. Guarino, S. Zeppetelli, P. Maddalena, A. Ambrosio, L. Ambrosio, J. Funct. Biomater. 11(1), 8 (2020)

31. E. Vaselli, C. Fedele, S. Cavalli, P.A. Netti, ChemPlusChem 80, 1547 (2015)

32. C. Rianna, A. Calabuig, M. Ventre, S. Cavalli, V. Pagliarulo, S. Grilli, P. Ferraro, P.A. Netti, A.C.S. Appl, Mater. Interfaces 7, 16984 (2015)

33. C. Rianna, L. Rossano, R.H. Kollarigowda, F. Formiggini, S. Cavalli, M. Ventre, P.A. Netti, Adv. Funct. Mater. 26, 7572 (2016)

34. M. Hendrikx, J. ter Schiphorst, E.P.A. van Heeswijk, G. Koçer, C. Knie, D. Bléger, S. Hecht, P. Jonkheijm, D.J. Broer, A.P. Schenning, Small 14, 1803274 (2018)

35. L. Rossano, C. Cimmino, S. Cavalli, M. Ventre, P.A. Netti, Adv. Mater. Interfaces 5, 1800890 (2018)

36. A. Puliafito, S. Ricciardi, F. Pirani, V. Čermochová, L. Boarino, N. De Leo, L. Primo, E. Descrovi, Adv. Sci. 6, 1801826 (2019)

37. G.S. Hartley, J. Chem. Soc. 113, 633 (1938)

38. G.C. Hampson, J. Monteath Robertson, J. Chem. Soc. (Resumed) 78, 409 (1941)

39. K.G. Yager, C.J. Barrett, J. Photochem. Photobiol. A Chem. 182, 250 (2006)

40. H. Rau, Photochemistry and Photophysics, vol. 2 (CRC, Boca Raton, 1990), pp. 119-141

41. A. Goulet-Hanssens, C.J. Barrett, J. Polym. Sci. A Polym. Chem. 51, 3058 (2013)

42. A. Natansohn, P. Rochon, M. Pezolet, P. Audet, D. Brown, S. To, Macromolecules 27, 2580 (1994)

43. D. Brown, A. Natansohn, P. Rochon, Macromolecules 28, 6116 (1995)

44. T. Ikeda, O. Tsutsumi, Science 268, 1873 (1995)

45. T. Todorov, L. Nikolova, N. Tomova, Appl. Opt. 23, 4588 (1984)

46. R. Lausten, P. Rochon, M. Ivanov, P. Cheben, S. Janz, P. Desjardins, J. Ripmeester, T. Siebert, A. Stolow, Appl. Opt. 44, 7039 (2005)

47. O.V. Yaroshchuk, A.D. Kiselev, Y. Zakrevskyy, T. Bidna, J. Kelly, L.-C. Chien, J. Lindau, Phys. Rev. E Stat. Nonlinear Soft Matter Phys. 68, 011803 (2003)

48. B.S. Neporent, O.V. Stolbova, Opt. Spektrosk. 14, 624 (1963)

49. M. Eich, Makromol. Chem. Rapid Commun. 8, 59 (1987)

50. A. Natansohn, S. Xie, P. Rochon, Macromolecules 25, 5531 (1992)

51. N.C. Holme, P.S. Ramanujam, S. Hvilsted, Appl. Opt. 35, 4622 (1996)

52. A. Petry, S. Kummer, H. Anneser, F. Feiner, C. Bräuchle, Ber. Bunsenges. Phys. Chem 97, 1281 (1993)

53. L.M. Blinov, M.V. Kozlovsky, M. Ozaki, K. Skarp, K. Yoshino, J. Appl. Phys. 84, 3860 (1998)

54. O. Yaroshchuk, T. Sergan, J. Lindau, S.N. Lee, J. Kelly, L.C. Chien, Mol. Cryst. Liq Cryst. Sci. Technol. Sect. A 359, 301 (2001)

55. L. Nikolova, T. Todorov, M. Ivanov, F. Andruzzi, S. Hvilsted, P.S. Ramanujam, Opt. Mater. 8, 255 (1997)

56. R.M. Tejedor, L. Oriol, J.L. Serrano, T. Sierra, J. Mater. Chem. 18, 2899 (2008)

57. L. Angiolini, T. Benelli, R. Bozio, A. Daurù, L. Giorgini, D. Pedron, Synth. Met. 139, 743 (2003)

58. J. Barberá, L. Giorgini, F. Paris, E. Salatelli, R.M. Tejedor, L. Angiolini, Chem. Eur. J. 14, 11209 (2008)

59. Z. Sekkat, W. Knoll, Photoreactive Organic Thin Films (Elsevier, Amsterdam, 2002).

60. S. Lee, H.S. Kang, J.-K. Park, Adv. Mater. 24, 2062 (2012)

61. Z. Mahimwalla, K.G. Yager, J.-I. Mamiya, A. Shishido, A. Priimagi, C.J. Barrett, Polym. Bull. 69, 967 (2012)

62. M. Saphiannikova, V. Toshchevikov, J. Ilnytskyi, Nonlinear Opt. Quantum Opt. 41, 27 (2010)

63. P. Karageorgiev, D. Neher, B. Schulz, B. Stiller, U. Pietsch, M. Giersig, L. Brehmer, Nat. Mater. 4, 699 (2005)

64. N.S. Yadavalli, S. Loebner, T. Papke, E. Sava, N. Hurduc, S. Santer, Soft Matter 12, 2593 (2016)

65. K.G. Yager, C.J. Barrett, in Polymeric nanostructures and their applications, ed. by H. S. Nalwa (Hardcover, 2006), pp. 1-38

66. P. Weis, W. Tian, S. Wu, Chem. Eur. J. 24, 6494 (2018)

67. J. Vapaavuori, A. Laventure, C.G. Bazuin, O. Lebel, C. Pellerin, J. Am. Chem. Soc. 137, 13510 (2015)

68. W. Xu, S. Sun, S. Wu, Angew. Chem. Int. Ed. 58, 9712 (2019) 
69. L. Rocha, V. Dumarcher, C. Denis, P. Raimond, C. Fiorini, J.M. Nunzi, J. Appl. Phys. 89(5), 3067 (2001)

70. J. Vapaavuori, C. Geraldine Bazuin, A. Priimagi, J. Mater. Chem. 6, 2168 (2018)

71. L. Nikolova, T. Todorov, Opt. Acta Int. J. Opt. 31, 579 (1984)

72. M. Eich, J.H. Wendorff, B. Reck, H. Ringsdorf, Makromol. Chem. Rapid Commun. 8, 59 (1987)

73. A. Natansohn, P. Rochon, J. Gosselin, S. Xie, Macromolecules 25, 2268 (1992)

74. D.Y. Kim, S.K. Tripathy, L. Li, J. Kumar, Appl. Phys. Lett. 66(10), 1166-1168 (1995)

75. N.K. Viswanathan, D.Y. Kim, S. Bian, J. Williams, W. Liu, L. Li, L. Samuelson, J. Kumar, S.K. Tripathy, J. Mater. Chem. 9, 1941 (1999)

76. K.G. Yager, C.J. Barrett, Curr. Opin. Solid State Mater. Sci. 5, 487 (2001)

77. C.J. Barrett, A.L. Natansohn, P.L. Rochon, J. Phys. Chem. 100, 8836 (1996)

78. N. Zettsu, T. Ogasawara, N. Mizoshita, S. Nagano, T. Seki, Adv. Mater. 20, 516 (2008)

79. J. Isayama, S. Nagano, T. Seki, Macromolecules 43, 4105 (2010)

80. J.E. Koskela, J. Vapaavuori, R.H.A. Ras, A. Priimagi, ACS Macro Lett. 3, 1196 (2014)

81. I. Kitamura, K. Kato, R.B. Berk, T. Nakai, M. Hara, S. Nagano, T. Seki, Sci. Rep. 10, 1 (2020)

82. A. Priimagi, A. Shevchenko, J. Polym. Sci. Part B Polym. Phys. 52, 163 (2014)

83. N. Hurduc, B.C. Donose, A. Macovei, C. Paius, C. Ibanescu, D. Scutaru, M. Hamel, N. BranzaNichita, L. Rocha, Soft Matter 10, 4640 (2014)

84. D.Y. Kim, L. Li, X.L. Jiang, V. Shivshankar, J. Kumar, S.K. Tripathy, Macromolecules 28, 8835 (1995)

85. A. Natansohn, P. Rochon, Adv. Mater. 11, 1387 (1999)

86. J. Paterson, A. Natansohn, P. Rochon, C.L. Callender, L. Robitaille, Appl. Phys. Lett. 69, 3318 (1996)

87. P. Rochon, A. Natansohn, C.L. Callender, L. Robitaille, Appl. Phys. Lett. 71, 1008 (1997)

88. R.J. Stockermans, P.L. Rochon, Appl. Opt. 38, 3714 (1999)

89. J.-W. Kang, M.-J. Kim, J.-P. Kim, S.-J. Yoo, J.-S. Lee, D.Y. Kim, J.-J. Kim, Appl. Phys. Lett. 82, 3823 (2003)

90. A. Perschke, T. Fuhrmann, Adv. Mater. 14, 841 (2002)

91. R. Windisch, P. Heremans, A. Knobloch, P. Kiesel, G.H. Döhler, B. Dutta, G. Borghs, Appl. Phys. Lett. 74, 2256 (1999)

92. W.H. Koo, S.M. Jeong, F. Araoka, K. Ishikawa, Nat. Photonics 4(4), 222 (2010)

93. P.S. Ramanujam, M. Pedersen, S. Hvilsted, Appl. Phys. Lett. 74, 3227 (1999)

94. L.P. Lee, R. Szema, Science 310, 1148 (2005)

95. H. Kusumaatmaja, R.J. Vrancken, C.W.M. Bastiaansen, J.M. Yeomans, Langmuir 24, 7299 (2008)

96. Y. Zhao, Q. Lu, M. Li, X. Li, Langmuir 23, 6212 (2007)

97. B. Liu, M. Wang, Y. He, X. Wang, Langmuir 22, 7405 (2006)

98. S. Yang, K. Yang, L. Niu, R. Nagarajan, S. Bian, A.K. Jain, J. Kumar, Adv. Mater. 16, 693 (2004)

99. J.H. Park, K.J. Park, T. Jiang, Q. Sun, J.-H. Huh, Z.L. Wang, S. Lee, J.H. Cho, Nano Energy 38, 412 (2017)

100. Y. Xia, E. Kim, X.-M. Zhao, J.A. Rogers, M. Prentiss, G.M. Whitesides, Science 273, 347 (1996)

101. C. Cocoyer, L. Rocha, C. Fiorini-Debuisschert, L. Sicot, D. Vaufrey, C. Sentein, B. Geffroy, P. Raimond, Thin Solid Films 511-512, 517 (2006)

102. C. Cocoyer, L. Rocha, L. Sicot, B. Geffroy, R. de Bettignies, C. Sentein, C. Fiorini-Debuisschert, P. Raimond, Appl. Phys. Lett. 88, 133108 (2006)

103. S.-I. Na, S.-S. Kim, S.-S. Kwon, J. Jo, J. Kim, T. Lee, D.-Y. Kim, Appl. Phys. Lett. 91,173509 (2007)

104. S.-I. Na, S.-S. Kim, J. Jo, S.-H. Oh, J. Kim, D.-Y. Kim, Adv. Funct. Mater. 18, 3956 (2008)

105. J. Kim, H.-J. Park, S.-I. Na, Y.-Y. Noh, D.-Y. Kim, Jpn. J. Appl. Phys. 53, 08NF02 (2014)

106. M. Kim, B. Kang, S. Yang, C. Drew, L.A. Samuelson, J. Kumar, Adv. Mater. 18, 1622 (2006)

107. S. Lee, J. Shin, Y.-H. Lee, S. Fan, J.-K. Park, Nano Lett. 10, 296 (2010)

108. S. Bian, L. Li, J. Kumar, D.Y. Kim, J. Williams, S.K. Tripathy, Appl. Phys. Lett. 73, 1817 (1998)

109. S. Bian, J.M. Williams, D.Y. Kim, L. Li, S. Balasubramanian, J. Kumar, S. Tripathy, J. Appl. Phys. 86, 4498 (1999)

110. H. Ishitobi, M. Tanabe, Z. Sekkat, S. Kawata, Opt. Express 15, 652 (2007)

111. H. Ishitobi, S. Shoji, T. Hiramatsu, H.-B. Sun, Z. Sekkat, S. Kawata, Opt. Express 16, 14106 (2008)

112. C. Rianna, L. Rossano, R.H. Kollarigowda, F. Formiggini, S. Cavalli, M. Ventre, P.A. Netti, Adv. Funct. Mater. 26, 7743 (2016)

113. A. Ambrosio, A. Camposeo, A. Carella, F. Borbone, D. Pisignano, A. Roviello, P. Maddalena, J. Appl. Phys. 107, 083110 (2010) 
114. C. Fedele, M. De Gregorio, P.A. Netti, S. Cavalli, C. Attanasio, Acta Biomater. 63, 317 (2017)

115. S. De Martino, S. Cavalli, P.A. Netti, Adv. Healthc. Mater. 9, 2000470 (2020)

116. N. Tsutsumi, A. Fujihara, Appl. Phys. Lett. 85, 4582 (2004)

117. C. Hubert, C. Fiorini-Debuisschert, I. Hassiaoui, L. Rocha, P. Raimond, J.-M. Nunzi, Appl. Phys. Lett. 87, 191105 (2005)

118. R. Barille, J.-M. Nunzi, S. Ahmadi-Kandjani, E. Ortyl, S. Kucharski, Opt. Commun. 280, 217 (2007)

119. C. Hubert, C. Fiorini-Debuisschert, L. Rocha, P. Raimond, J.-M. Nunzi, J. Opt. Soc. Am. B 24, 1839 (2007)

120. S. Lee, Y.-C. Jeong, J.-K. Park, Appl. Phys. Lett. 93, 031912 (2008)

121. H. Leblond, R. Barille, S. Ahmadi-Kandjani, J.-M. Nunzi, E. Ortyl, S. Kucharski, J. Phys. B At. Mol. Opt. Phys. 42, 205401 (2009)

122. J. Yin, G. Ye, X. Wang, Langmuir 26, 6755 (2010)

123. A. Ambrosio, P. Maddalena, A. Carella, F. Borbone, A. Roviello, M. Polo, A.A.R. Neves, A. Camposeo, D. Pisignano, J. Phys. Chem. C 115, 13566 (2011)

124. X. Wang, J. Yin, X. Wang, Macromolecules 44, 6856 (2011)

125. X. Wang, J. Yin, X. Wang, Langmuir 27, 12666 (2011)

126. A. Ambrosio, S. Girardo, A. Camposeo, D. Pisignano, P. Maddalena, Appl. Phys. Lett. 102, 093102 (2013)

127. L. Mazaheri, S. Ahmadi-Kandjani, J.-M. Nunzi, Opt. Commun. 298-299, 150 (2013)

128. H. Galinski, A. Ambrosio, P. Maddalena, I. Schenker, R. Spolenak, F. Capasso, Proc. Natl. Acad. Sci. 111, 17017 (2014)

129. A. Sobolewska, S. Bartkiewicz, J. Mater. Chem. C 3, 5616 (2015)

130. V. Teboul, R. Barillé, P. Tajalli, S. Ahmadi-Kandjani, H. Tajalli, S. Zielinska, E. Ortyl, Soft Matter 11(32), 6444 (2015)

131. L. Mazaheri, S.R. Bobbara, O. Lebel, J.-M. Nunzi, Opt. Lett. 41, 2958 (2016)

132. J. Noga, A. Sobolewska, S. Bartkiewicz, M. Virkki, A. Priimagi, Macromol. Mater. Eng. 302, 1600329 (2017)

133. C. Hubert, C. Fiorini-Debuisschert, P. Raimond, J.-M. Nunzi, Organic Photonic Mater. Dev. 4991, 313 (2003)

134. Y. Gritsai, L.M. Goldenberg, J. Stumpe, Opt. Express 19, 18687 (2011)

135. H.S. Kang, S. Lee, S.-A. Lee, J.-K. Park, Adv. Mater. 25, 5490 (2013)

136. S. Lee, H.S. Kang, J.-K. Park, Adv. Funct. Mater. 21, 1769 (2011)

137. A.M. Dubrovkin, R. Barillé, E. Ortyl, S. Zielinska, Chem. Phys. Lett. 599, 7 (2014)

138. A.M. Dubrovkin, R. Barillé, E. Ortyl, S. Zielinska, Opt. Commun. 334, 147 (2015)

139. W. Wang, C. Du, X. Wang, X. He, J. Lin, L. Li, S. Lin, Angew. Chem. Int. Ed Engl. 53, 12116 (2014)

140. S.G. Sorkhabi, R. Barille, S. Ahmadi-Kandjani, S. Zielinska, E. Ortyl, Opt. Mater. 66, 573 (2017)

141. W. Wang, Y. Yao, T. Luo, L. Chen, J. Lin, L. Li, S. Lin, A.C.S. Appl, Mater. Interfaces 9, 4223 (2017)

142. B. Liu, Y. He, P. Fan, X. Wang, Langmuir 23, 11266 (2007)

143. X. Kong, X. Wang, T. Luo, Y. Yao, L. Li, S. Lin, A.C.S. Appl, Mater. Interfaces 9, 19345 (2017)

144. S. Lee, H.S. Kang, A. Ambrosio, J.-K. Park, L. Marrucci, ACS Appl. Mater. Interfaces. 7, 8209 (2015)

145. H.S. Kang, S. Lee, J.-K. Park, Adv. Funct. Mater. 21, 4412 (2011)

146. S. Lee, H.S. Kang, J.-K. Park, Adv. Mater. 24, 2069 (2012)

147. F. Pirani, A. Angelini, S. Ricciardi, F. Frascella, E. Descrovi, Appl. Phys. Lett. 110, 101603 (2017)

148. T. Ikeda, S. Horiuchi, D.B. Karanjit, S. Kurihara, S. Tazuke, Macromolecules 23, 36 (1990)

149. C.J. Barrett, J. Mamiya, K.G. Yager, T. Ikeda, Soft Matter 3(10), 1249-1261 (2007)

150. M. Hendrikx, A.P.H.J. Schenning, M.G. Debije, D.J. Broer, Crystals 7, 231 (2017)

151. T.J. White, D.J. Broer, Nat. Mater. 14, 1087 (2015)

152. S. Tazuke, S. Kurihara, T. Ikeda, Chem. Lett. 16, 911 (1987)

153. O. Tsutsumi, Y. Demachi, A. Kanazawa, T. Shiono, T. Ikeda, Y. Nagase, J. Phys. Chem. B 102, 2869 (1998)

154. T. Ikeda, M. Nakano, Y. Yu, O. Tsutsumi, A. Kanazawa, Adv. Mater. 15, 201 (2003)

155. X. Qing, L. Qin, W. Gu, Y. Yu, Liq. Cryst. 43, 2114 (2016)

156. P.M. Hogan, A.R. Tajbakhsh, E.M. Terentjev, Phys. Rev. E Stat. Nonlinear Soft Matter Phys. 65, 041720 (2002)

157. T. Ikeda, S. Horiuchi, D.B. Karanjit, S. Kurihara, S. Tazuke, Chem. Lett. 17, 1679 (1988)

158. H. Finkelmann, E. Nishikawa, G.G. Pereira, M. Warner, Phys. Rev. Lett. 87, 015501 (2001)

159. M.-H. Li, P. Keller, B. Li, X. Wang, M. Brunet, Adv. Mater. 15, 569 (2003) 
160. Y. Yu, M. Nakano, T. Ikeda, Nature 425, 145 (2003)

161. R. Yin, W. Xu, M. Kondo, C.-C. Yen, J.-I. Mamiya, T. Ikeda, Y. Yu, J. Mater. Chem. 19, 3141 (2009)

162. T. Ube, T. Ikeda, Angew. Chem. Int. Ed. 53, 10290 (2014)

163. Y. Yu, M. Nakano, A. Shishido, T. Shiono, T. Ikeda, Chem. Mater. 16, 1637 (2004)

164. F. Lancia, A. Ryabchun, A.-D. Nguindjel, S. Kwangmettatam, N. Katsonis, Nat. Commun. 10(1), $1-8(2019)$

165. Y. Yu, T. Maeda, J.-I. Mamiya, T. Ikeda, Angew. Chem. Int. Ed. 46, 881 (2007)

166. H. Jiang, C. Li, X. Huang, Nanoscale 5, 5225 (2013)

167. H. Wermter, H. Finkelmann, E-polymers 1, 013 (2001)

168. H. Zeng, P. Wasylczyk, C. Parmeggiani, D. Martella, M. Burresi, D.S. Wiersma, Adv. Mater. 27, 3883 (2015)

169. D. Martella, S. Nocentini, D. Nuzhdin, C. Parmeggiani, D.S. Wiersma, Adv. Mater. 29, 1704047 (2017)

170. Y. Zhang, J. Xu, F. Cheng, R. Yin, C.C. Yen, J. Mater. Chem. 20(34), 7123 (2010)

171. M. Yamada, M. Kondo, R. Miyasato, Y. Naka, J.-I. Mamiya, M. Kinoshita, A. Shishido, Y. Yu, C.J. Barrett, T. Ikeda, J. Mater. Chem. 19, 60 (2009)

172. K.D. Harris, R. Cuypers, P. Scheibe, C.L. van Oosten, C.W.M. Bastiaansen, J. Lub, D.J. Broer, J. Mater. Chem. 15, 5043 (2005)

173. C.L. van Oosten, K.D. Harris, C.W.M. Bastiaansen, D.J. Broer, Eur. Phys. J. E Soft Matter 23, 329 (2007)

174. S. Iamsaard, S.J. Aßhoff, B. Matt, T. Kudernac, J.J.L.M. Cornelissen, S.P. Fletcher, N. Katsonis, Nat. Chem. 6, 229 (2014)

175. A. Priimagi, A. Shimamura, M. Kondo, T. Hiraoka, S. Kubo, J.-I. Mamiya, M. Kinoshita, T. Ikeda, A. Shishido, ACS Macro Lett. 1, 96 (2012)

176. D. Liu, C.W.M. Bastiaansen, J.M.J. den Toonder, D.J. Broer, Macromolecules 45, 8005 (2012)

177. D. Liu, C.W.M. Bastiaansen, J.M.J. den Toonder, D.J. Broer, Angew. Chem. Int. Ed. 51, 892 (2012)

178. A. Dillow, A. Lowman, Biomimetic Materials and Design: Biointerfacial Strategies, Tissue Engineering and Targeted Drug Delivery (CRC Press, Boca Raton, 2002).

179. M. Ventre, F. Causa, P.A. Netti, J.R. Soc, Interface 9, 2017 (2012)

180. C. Frantz, K.M. Stewart, V.M. Weaver, J. Cell Sci. 123, 4195 (2010)

181. M. Guvendiren, J.A. Burdick, Adv. Healthc. Mater. 2, 155 (2013)

182. T. Gong, K. Zhao, G. Yang, J. Li, H. Chen, Y. Chen, S. Zhou, Adv. Healthc. Mater. 3, 1608 (2014)

183. D.M. Le, K. Kulangara, A.F. Adler, K.W. Leong, V.S. Ashby, Adv. Mater. 23, 3278 (2011)

184. G. Koçer, J. Ter Schiphorst, M. Hendrikx, H.G. Kassa, P. Leclère, A.P.H.J. Schenning, P. Jonkheijm, Adv. Mater. 29, 27 (2017)

185. M. Hendrikx, J. ter Schiphorst, E.P.A. van Heeswijk, G. Koçer, C. Knie, D. Bléger, S. Hecht, P. Jonkheijm, D.J. Broer, P.H. Albertus, Small 14, 1870240 (2018)

186. I. Tomatsu, K. Peng, A. Kros, Adv. Drug Deliv. Rev. 63, 1257 (2011)

187. F.A. Pennacchio, C. Fedele, S. De Martino, S. Cavalli, R. Vecchione, P.A. Netti, A.C.S. Appl, Mater. Interfaces 10, 91 (2018)

188. A.M. Rosales, K.M. Mabry, E.M. Nehls, K.S. Anseth, Biomacromol 16, 798 (2015)

189. I.-N. Lee, O. Dobre, D. Richards, C. Ballestrem, J.M. Curran, J.A. Hunt, S.M. Richardson, J. Swift, L.S. Wong, A.C.S. Appl, Mater. Interfaces 10, 7765 (2018)

190. L. Qin, Y. Yu, in Responsive Polymer Surfaces: Dynamics in Surface Topography, ed. by D. Liu, D. J. Broer (Wiley-VCH, 2017), pp. 1-34 